\title{
Distribution, Amplitude, Incidence, Co-Occurrence, and Propagation of Human K-Complexes in Focal Transcortical Recordings ${ }^{1,2,3}$
}

\author{
Rachel A. Mak-McCully, ${ }^{1}$ Burke Q. Rosen, ${ }^{2}$ Matthieu Rolland, ${ }^{2}$ Jean Régis, ${ }^{4,5,6}$ Fabrice Bartolomei, ${ }^{4,5,6}$ \\ Marc Rey, ${ }^{4,5,6}$ Patrick Chauvel ${ }^{4,5,6, *}$, Sydney S. Cash, ${ }^{7, *}$ and Eric Halgren ${ }^{1,2,3, *}$
}

\section{DOI:http://dx.doi.org/10.1523/ENEURO.0028-15.2015}

${ }^{1}$ Department of Neurosciences, University of California, San Diego, San Diego, California 92093, ${ }^{2}$ Department of Radiology, University of California, San Diego, San Diego, California 92093, ${ }^{3}$ Department of Psychiatry, University of California, San Diego, San Diego, California 92093, ${ }^{4}$ Aix-Marseille Université, Marseille 13385, France, ${ }^{5}$ INSERM, Institut de Neurosciences des Systèmes UMR 1106, Marseille 13005, France, ${ }^{6}$ Assistance Publique-Hôpitaux de Marseille, Timone Hospital, Marseille 13005, France, ${ }^{7}$ Department of Neurology, Massachusetts General Hospital and Harvard Medical School, Harvard University, Boston, Massachusetts 02114

\begin{abstract}
$\mathrm{K}$-complexes (KCs) are thought to play a key role in sleep homeostasis and memory consolidation; however, their generation and propagation remain unclear. The commonly held view from scalp EEG findings is that KCs are primarily generated in medial frontal cortex and propagate parietally, whereas an electrocorticography (ECOG) study suggested dorsolateral prefrontal generators and an absence of KCs in many areas. In order to resolve these differing views, we used unambiguously focal bipolar depth electrode recordings in patients with intractable epilepsy to investigate spatiotemporal relationships of human KCs. KCs were marked manually on each channel, and local generation was confirmed with decreased gamma power. In most cases (76\%), KCs occurred in a single location, and rarely (1\%) in all locations. However, if automatically detected KC-like phenomena were included, only $15 \%$ occurred in a single location, and $27 \%$ occurred in all recorded locations. Locally generated KCs were found in all sampled areas, including cingulate, ventral temporal, and occipital cortices. Surprisingly, KCs were smallest and occurred least frequently in anterior prefrontal channels. When KCs occur on two channels, their peak order is consistent in only $13 \%$ of cases, usually from prefrontal to lateral temporal. Overall, the anteriorposterior separation of electrode pairs explained only $2 \%$ of the variance in their latencies. KCs in stages 2 and 3 had similar characteristics. These results open a novel view where KCs overall are universal cortical phenomena, but each KC may variably involve small or large cortical regions and spread in variable directions, allowing flexible and heterogeneous contributions to sleep homeostasis and memory consolidation.
\end{abstract}

Key words: depth recordings; k-complex; memory; SEEG; sleep

\section{Significance Statement}

This is the first examination of the location of K-complexes (KCs) and their temporal relationship across the cortex using such focal measures of brain activity. KCs, along with sleep spindles and slow oscillations, are thought to play important roles in the restorative and memory consolidation processes of non-rapid eye movement sleep. KCs are unique from these other markers because they indicate isolated periods of cortical silence. We describe here that $\mathrm{KCs}$ occur in parts of the brain previously thought not to generate KCs. We show they may co-occur over small or large parts of the cortex, but do not propagate in a systematic way. This variability could reflect and support consolidation processes devoted to memories involving the corresponding cortical areas and sequences. 


\section{Introduction}

$\mathrm{K}$-complexes (KCs) are isolated down states occurring both spontaneously and in response to sensory stimuli during non-rapid eye movement (NREM) sleep (Colrain, 2005; Cash et al., 2009). Here we examine the spatial distribution and temporal dynamics of KCs across the cortex using bipolar stereoencephalographic (SEEG) recordings to address basic questions that are unanswered or ambiguous in the $\mathrm{KC}$ literature. Which cortical regions generate KCs? How variable is KC amplitude? How variable is the KC occurrence rate? How often do KCs cooccur across the cortex? Do KCs propagate sequentially from one cortical location to another?

Answers to some of these questions have varied due to the recording technique. Scalp EEG examination of the $\mathrm{KC}$ indicates that $\mathrm{KCs}$ are largest in amplitude and most frequent over midline frontal sites (Colrain, 2005; Halász, 2005). Cortical surface electrocorticography (ECOG) recordings and average reference SEEG recordings have led to the conclusion that many cortical areas crucial for memory do not generate KCs (Wennberg, 2010). Scalp EEG and ECOG recordings, however, are difficult to interpret due to potential overlap and cancellation from multiple distributed generators. Additionally, average references may detect signals that are not locally generated.

Bipolar SEEG recordings eliminate the ambiguity of scalp EEG, ECOG, and average reference SEEG. In both scalp EEG and ECOG, individual electrodes record activity from local and distant generators, which are difficult to disentangle; scalp EEG is further smeared by the skull. In bipolar SEEG recordings, nearby contacts along an electrode are subtracted from one another. Adjacent contacts chosen for analysis can have one contact just above the gray matter in the CSF, and one just below the gray matter in the white matter. This subtraction provides a focal recording of brain activity unavailable with scalp EEG or

Received April 2, 2015; accepted July 24, 2015; First published September 02, 2015.

${ }^{1}$ The authors declare no competing financial interests.

${ }^{2}$ Author Contributions: R.M.-M., P.C., S.S.C., and E.H. designed research; R.M.-M., B.Q.R., S.S.C., and E.H. performed research; BQR, M. Rolland, J.R., F.B., and P.C. contributed unpublished reagents/analytic tools; R.M.-M., B.Q.R., M. Rolland, J.R., M. Rey, S.S.C.; E.H. analyzed data; R.M.-M. and E.H. wrote the paper.

${ }^{3}$ This study is based upon work that was supported by National Institutes of Health Grant R01-MH-099645, Multidisciplinary University Research Initiative Grant ONR N00014-13-1-0672, the National Science Foundation Graduate Research Fellowship, and the Chateaubriand Fellowship.

${ }^{\star}$ P.C., S.S.C., and E.H. are co-senior authors.

Acknowledgements: We dedicate this work to Frederic Sauvaget, an exceptional neurosurgeon, neuroscientist, and colleague. His passion for his work was truly inspirational. In addition, we thank neurosurgeons Romain Carron, Didier Scavarda, and Emad Eskandar for patient implantations. We especially thank Catherine Liegeois-Chauvel for assistance in electrode localizations, facilitating research access, and scientific support.

Correspondence should be addressed to Rachel A. Mak-McCully, Multimodal Imaging Laboratory, UC San Diego, 9500 Gilman Drive, Mail Code 0841, La Jolla, CA 92093. E-mail: rmakmccu@ucsd.edu.

DOI:http://dx.doi.org/10.1523/ENEURO.0028-15.2015

Copyright (C) 2015 Mak-McCully et al.

This is an open-access article distributed under the terms of the Creative Commons Attribution 4.0 International, which permits unrestricted use, distribution and reproduction in any medium provided that the original work is properly attributed.
ECOG. Even the use of an average reference in SEEG may mask or augment a local signal, which is not the case when SEEG is analyzed using bipolar subtraction. The ambiguity inherent in scalp EEG, ECOG, and referential SEEG may confound interpretations of both where KCs are generated and how they co-occur or spread across the cortex.

Another source of ambiguity in previous studies is the grouping together of $\mathrm{KCs}$ and slow oscillations (SOs), which occur as a series of up and down states in NREM stage 3 sleep (N3). SOs and KCs share a frequency of $\sim 1$ $\mathrm{Hz}$, and the $\mathrm{KC}$ is identical to the down state of the SO (Cash et al., 2009). The commonly used automated SO detection methods identify all large low-frequency deflections, including KCs. Scalp EEG recordings have led to the interpretation that SOs propagate across the cortex in an anterior-to-posterior direction, with a delay of $\sim 200 \mathrm{~ms}$ (Massimini et al., 2004; Murphy et al., 2009). Since these studies included KCs as well as SOs in their analyses, these studies provide insight into the propagation of $\mathrm{KCs}$ as well. A major distinction between SOs and KCs is that the $\mathrm{KC}$ is an isolated event: it is neither part of an ongoing oscillation nor is preceded by an up state. It is, therefore, possible to pinpoint the start of a $\mathrm{KC}$ in the cortex. In contrast, because the $\mathrm{SO}$ is an oscillation, it can be difficult to determine which waves correspond to each other across different cortical areas, resulting in ambiguous order and dependence.

By rigorously quantifying the spatial distribution and temporal dynamics of KCs across the cortex, we focused on three questions. First, are KCs a fundamental neocortical state that are locally generated in all neocortical areas with similar amplitudes and rates of occurrence? Particular attention was paid to the cingulate, ventral temporal, and occipital cortices where Wennberg (2010) failed to find $\mathrm{KC}$ generators using intracranial recordings. Similarly, special attention was paid to lateral cortex, in general, where other studies using high-density EEG failed to find generators of evoked KCs or isolated SOs (i.e., KCs; Murphy et al., 2009; Riedner et al., 2011). Second, do KCs co-occur in multiple lobes and both hemispheres, or do they occur in a more isolated fashion? Third, do KCs systematically propagate from anterior to posterior locations, and in particular from prefrontal to parietal?

The three NREM sleep markers-sleep spindles, SOs, and $\mathrm{KCs}$ - have all been implicated in the restorative and memory processes that occur during sleep (Diekelmann and Born, 2010; Tononi and Cirelli, 2014). By understanding where the $\mathrm{KC}$ occurs in the brain, how the $\mathrm{KC}$ occurs temporally across the cortex, and whether it is an event that may manifest heterogeneously each time it occurs, we can understand how the role of each generating structure and their orchestration lead to sleep homeostasis and memory consolidation.

\section{Materials and Methods}

SEEG recordings were obtained in nine patients (eight women, one man; mean age, $41.4 \pm 4.9$ years) who experienced pharmacoresistant epilepsy to localize their 
Table 1. Demographic and clinical information for all patients studied

\begin{tabular}{|c|c|c|c|c|c|c|c|c|}
\hline Subject & Gender & $\begin{array}{l}\text { Age } \\
\text { (years) }\end{array}$ & Handedness & $\begin{array}{l}\text { Clinical } \\
\text { diagnosis }\end{array}$ & $\begin{array}{l}\text { Pathologic } \\
\text { diagnosis }\end{array}$ & Imaging & Focus & $\begin{array}{l}\text { IQ } \\
\text { (FSIQ) }\end{array}$ \\
\hline 1 & M & 45 & L & CPS; bitemporal & $\begin{array}{l}\text { No pathology } \\
\text { obtained }\end{array}$ & & $\begin{array}{l}\text { Left and right mesial } \\
\text { temporal lobes }\end{array}$ & 83 \\
\hline 2 & $\mathrm{~F}$ & 58 & $\mathrm{R}$ & $\begin{array}{l}\text { CPS; possible generalized } \\
\text { epilepsy }\end{array}$ & $\begin{array}{l}\text { No pathology } \\
\text { obtained }\end{array}$ & & Likely generalized & 80 \\
\hline 3 & $\mathrm{~F}$ & 45 & $\mathrm{R}$ & CPS; multifocal & $\begin{array}{c}\text { Multifocal: temporal, } \\
\text { parietal, occipital }\end{array}$ & & Temporal & Average \\
\hline 4 & $\mathrm{~F}$ & 65 & $\mathrm{R}$ & $\begin{array}{l}\text { CPS; temporal lobe epilepsy } \\
\text { with two foci: left mesial } \\
\text { temporal structures } \\
\text { and right subfrontal } \\
\text { region }\end{array}$ & $\begin{array}{l}\text { No pathology } \\
\text { obtained }\end{array}$ & & $\begin{array}{l}\text { Right subfrontal and } \\
\text { anterior temporal }\end{array}$ & 101 \\
\hline 5 & $\mathrm{~F}$ & 27 & $\mathrm{R}$ & Right temporal lobe epilepsy & $\begin{array}{l}\text { No pathology } \\
\text { obtained }\end{array}$ & $\begin{array}{l}\text { Right hippocampal } \\
\text { sclerosis }\end{array}$ & $\begin{array}{l}\text { Hippocampus, entorhinal } \\
\text { cortex, amygdala }\end{array}$ & Average \\
\hline 6 & $\mathrm{~F}$ & 32 & $\mathrm{R}$ & $\begin{array}{l}\text { Left temporal } \\
\text { lobe epilepsy }\end{array}$ & $\begin{array}{l}\text { Non-specific } \\
\text { gliosis }\end{array}$ & Normal & $\begin{array}{l}\text { Hippocampus, entorhinal } \\
\text { cortex, amygdala }\end{array}$ & 77 \\
\hline 7 & $\mathrm{~F}$ & 23 & $\mathrm{R}$ & $\begin{array}{l}\text { Right temporal } \\
\text { lobe epilepsy }\end{array}$ & $\begin{array}{l}\text { Type I focal } \\
\text { dysplasia }\end{array}$ & Normal & $\begin{array}{l}\text { Hippocampus, entorhinal } \\
\text { cortex,anterior } \\
\text { insula }\end{array}$ & 86 \\
\hline 8 & $\mathrm{~F}$ & 28 & $\mathrm{R}$ & $\begin{array}{l}\text { Right temporal } \\
\text { occipital epilepsy }\end{array}$ & $\begin{array}{l}\text { Type II focal } \\
\text { dysplasia }\end{array}$ & $\begin{array}{l}\text { Focal cortical } \\
\text { dysplasia in right } \\
\text { fusiform }\end{array}$ & $\begin{array}{l}\text { Fusiform gyrus, } \\
\text { entorhinal } \\
\text { cortex }\end{array}$ & Average \\
\hline 9 & $\mathrm{~F}$ & 50 & $\mathrm{R}$ & $\begin{array}{l}\text { Right temporal } \\
\text { occipital epilepsy }\end{array}$ & $\begin{array}{l}\text { No pathology } \\
\text { obtained }\end{array}$ & Normal & $\begin{array}{l}\text { Right fusiform } \\
\text { gyrus }\end{array}$ & 110 \\
\hline
\end{tabular}

FSIQ, Full Scale Intelligence Quotient; CPS, Complex Partial Seizures.

seizure focus prior to possible resection at Massachusetts General Hospital or La Timone Hospital (Table 1). The anatomical nomenclature follows that of Destrieux et al. (2010). At Massachusetts General Hospital, electrodes were localized with respect to anatomical structures using computed tomography (CT) with the electrodes in place, and intraoperative photographs (Dykstra et al., 2012). Depth electrodes (SEEG) had either eight contacts with 5 $\mathrm{mm}$ center-to-center spacing or six contacts with $8 \mathrm{~mm}$ center-to-center spacing. Each contact was $2.4 \mathrm{~mm}$ long with a diameter of $1.28 \mathrm{~mm}$. The electrodes usually passed approximately perpendicular to the midsagittal plane. At La Timone Hospital, electrodes were localized using the fusion of CT scanning with implanted electrodes and MRI. In one case, surgical planning and preoperative MRI were used to localize the electrodes. SEEG electrodes had 10 or 15 contacts with $3.5 \mathrm{~mm}$ center-tocenter spacing; in some cases, the 15 contact electrodes contained three sets of five contacts, with $3.5 \mathrm{~mm}$ centerto-center spacing within the set and 7 or $11 \mathrm{~mm}$ between each set of five contacts. Contacts were $2 \mathrm{~mm}$ long with a $0.8 \mathrm{~mm}$ diameter. Electrodes were both perpendicular and oblique. Prior to surgery, fully informed consent was obtained under the auspices of local institutional review boards. The signals were sampled at $256,500,512$, or $1024 \mathrm{~Hz}$.

Adjacent contacts along each SEEG electrode were subtracted to create bipolar contacts. The bipolar derivation was obtained by subtracting the more lateral lead from the more medial adjacent lead. In the case where the electrode is passing through the most superficial cortex, the lateral lead is over the pia and the medial lead is in the underlying white matter. Since the KC is surface negative (Cash et al., 2009), a medial - lateral derivation would in this case result in a positive peak. In contrast, at the midline locations (e.g., the crown of the cingulate gyrus), the medial lead is above the pia and the lateral is in the underlying white matter, and consequently $\mathrm{KCs}$ are negative in the medial - lateral derivation. Intermediate bipolar pairs could be either polarity depending upon the exact relationship of the contacts to local cortical folding.

Contacts to include for analysis were chosen by examining both physiological and anatomical criteria. Physiologically, referential recordings from a given depth electrode were examined for successive contacts which recorded polarity-inverted spontaneous activity. In such cases, anatomically, one contact of the bipolar pair would typically lay in the CSF above the cortical gray matter, while the other lay just below it in the white matter. In the case of overlapping bipolar contacts (i.e., two pairs sharing a contact), only one bipolar pair was included for analysis. A total of 55 bipolar contacts were analyzed. This bipolar method is free of volume conduction and reference lead issues, obtaining unambiguously focal cortical recordings. Furthermore, to be included for analysis, contacts needed to be distant from the epileptogenic focus, free of epileptiform activity (spikes or pathological slowing), and exhibit KCs.

Usable scalp EEG channels were recorded in the four subjects from Massachusetts General Hospital, but not in the five subjects from La Timone Hospital. When present, the scalp recordings lacked the full montage used for formal polysomnography (Silber et al., 2007). Periods when patients were behaviorally noted to be sleeping were selected for analysis. As this study sought to characterize KCs as measured locally, rather than defined by scalp EEG, the initial selection of KCs was blind to sleep staging. As a secondary analysis, sleep staging based on a scalp EEG-as well as EMG and EOG, when availablefor subjects recorded at Massachusetts General Hospital, or based on the SEEG for subjects recorded at La Timone 
Hospital, was performed for all subjects by a qualified rater (M. Rey).

KCs were manually marked independently on each bipolar SEEG contact for each subject. Each night was considered independent and studied separately. Selected KCs were isolated (i.e., they were not part of a preceding oscillation) and exhibited a multiphasic morphology, with a significant drop peaking at $\sim 500 \mathrm{~ms}$ from the beginning of the waveform, often followed by a rebound peaking at $\sim 900$ ms (Colrain, 2005). Each channel was then bandpassed from 0.1 to $5 \mathrm{~Hz}$ in order to select the peak of the manually marked KC.

To confirm that selected KCs, regardless of their polarity, were down states, we tested for a drop in high gamma power (HGP) at the time of the KC in all channels for each subject. If a subject had multiple nights, only one sample night was used to test an HGP drop in all channels. KCs were therefore not validated individually for a drop in HGP, but were validated over all KCs in each channel, for 1 night per subject. HGP was calculated from 60 to 120 $\mathrm{Hz}$, or from 70 to $120 \mathrm{~Hz}$, depending on whether the line noise was at 50 or $60 \mathrm{~Hz}$, respectively. Visually, a drop in HGP up to $120 \mathrm{~Hz}$ was determined by a time frequency analysis using default wavelets in EEGLAB (Delorme and Makeig, 2004): with the most negative peak of the $K C$ at time zero, -1.5 to $-0.5 \mathrm{~s}$ was used as a comparison baseline for a $t$ test at a $p<0.01$ uncorrected threshold. The drop in HGP seen at the time of the KC was further verified using a Hilbert transform applied to each KC. The analytic amplitude of the Hilbert transform was calculated after applying a bandpass filter for the appropriate HGP range (either $60-120 \mathrm{~Hz}$ or $70-120 \mathrm{~Hz}$ ) and averaged over all KCs for each channel. Compared to a baseline period from -1.5 to $-1 \mathrm{~s}$ prior to the most negative peak of the $\mathrm{KC}$ at time zero, periods of significant $(p<0.01, t$ test, false discovery rate corrected) drop in HGP were required for KC confirmation. These analyses were accomplished with custom MATLAB (2009) routines with dependencies including the FieldTrip (Oostenveld et al., 2011) toolbox.

In order to compare our bipolar derivations with the common average reference recordings used in a previous study (Wennberg, 2010), referential recordings were reconstructed for the individual leads used in a typical transcortical bipolar pair, and in nearby leads on the same probe but in the white matter. HGP was calculated as above (see Fig. 3 for details).

$\mathrm{KC}$ detections were grouped across channels to classify the number of unique $\mathrm{KC}$ events for each night for each subject using R (R Development Core Team, 2004). Starting with the first KC peak, additional channels were classified as participating in the same KC if their peak time was $\leq 200 \mathrm{~ms}$ from this first peak latency. The next KC peak after the $200 \mathrm{~ms}$ window was the beginning of the second $\mathrm{KC}$ event. The grouping of $\mathrm{KC}$ events continued until the last KC peak was classified. As a control, KCs were also regrouped using a $200 \mathrm{~ms}$ crawling window: after each KC peak detection, the detection window was shifted $200 \mathrm{~ms}$ to the right. The detection window continued shifting to the right until no more peaks were de- tected. These regroupings were not used for any of the analyses described below.

Although the $200 \mathrm{~ms}$ window procedure would allow KCs occurring in at least two channels to have a maximum peak difference up to $200 \mathrm{~ms}$, the actual maximum difference was observed to be much less. This latency difference was quantified by calculating the delay between the first channel peak and the last channel peak for each KC event. The mean and SD over all KCs for each night were then calculated.

To study how KC amplitude and KC occurrence rate may vary across the cortex, bipolar channels were grouped by anatomical locations into 10 groups (Fig. 1). To test KC amplitude, a linear mixed-effects model (Pinheiro et al.,2015) was performed. Location was used as a fixed effect. Placement of electrodes for each patient was chosen to localize the patient's epilepsy, but electrodes included in this analysis were not shown to be part of each patient's seizure focus. Therefore, patient and location were considered independent. Interpatient variability was adjusted for by using patient as a random effect. Channel variability due to electrode placement with respect to the surrounding cortex, as well as variable contact spacing, was taken into account by using channel as a random nested effect.

To test the KC occurrence rate, a linear mixed-effects model of the inter-KC interval was performed with location as a fixed effect and patient as a random effect. In both the amplitude and occurrence rate models, the orbitofrontal cortex group served as the reference. For the residuals of the model to be normally distributed, a log transform was applied to the amplitudes and a Box-Cox Transform (Box and Cox, 1964) was applied to the inter-KC intervals. The normality of the residuals was checked visually. All models were developed using the LME procedure implemented in the nlme package (Pinheiro et al., 2015) of R. Due to the limitations of this package, confidence intervals for all predicted values derived from the linear mixed-effects models could not be provided. In order to quantify the difference in either amplitude or occurrence rate between a tested location and the orbitofrontal reference, population level predictions were computed for each location. An inverse BoxCox transformation was then applied to these estimates to obtain values in either microvolts or milliseconds in order to compute a relative percentage to the reference level.

To test whether KCs co-occur in multiple channels, we compared the number of KC events observed in exactly one channel, versus more than one channel, to what those numbers would be by chance. Fisher's exact test was performed in $\mathrm{R}$ to determine whether the numbers significantly differed at $\alpha=0.05$. The number of KCs that would be expected in one channel or multiple channels by chance was estimated by randomizing the latencies between two consecutive KCs within each channel. This provided a new list of KC peaks for each channel. This randomization was performed 1000 times. These randomized latency differences were converted into latencies by adding in the latency of the first detected KC for each 
A

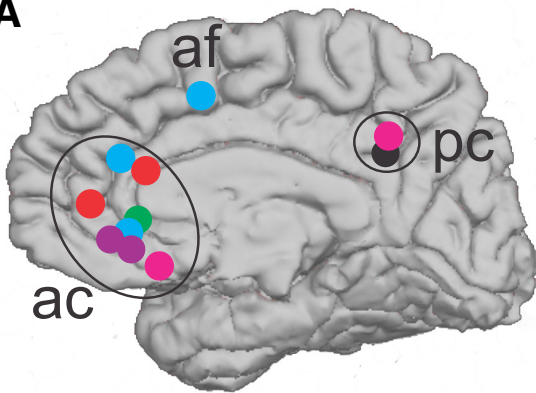

ac-anterior cingulate and subcallosal af-anterior prefrontal cortex pc-posterior cingulate pf-posterior prefrontal cortex cs-central sulcus in-insula f3op-inferior frontal gyrus; pars opercularis of-orbitofrontal cortex tp-temporo-parieto-occipital junction vt-ventrolateral temporal cortex

B

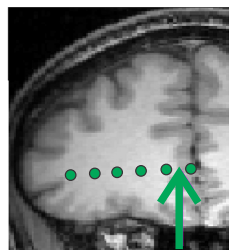

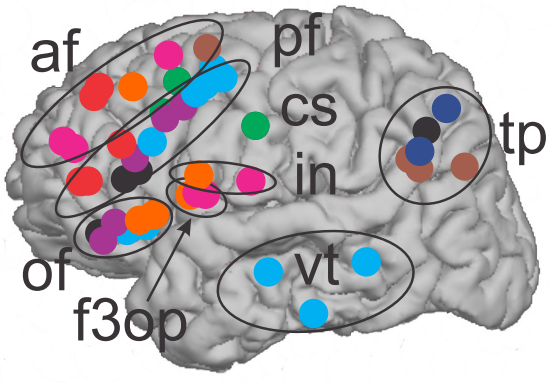

Subject \#1

Subject \#2

Subject \#3

Subject \#4

Subject \#5

Subject \#6

Subject \#7

Subject \#8

Subject \#9

Figure 1. Bipolar SEEG contacts. A, Bipolar SEEG contacts, color
lateral (right) surfaces of the brain. Bipolar SEEG contacts are gro
and/or one of the following labels: ac (anterior cingulate and sub
(posterior prefrontal cortex); cs (central sulcus); in (insula); f3op
(temporo-parieto-occipital junction); vt (ventrolateral temporal co
4 (purple) are superimposed on the subjects' MRls. The arrows
electrode. From the MRI, it is possible to see that the two contact
channel. Additionally, the latency of the first detected KC
was added back to keep the number of latencies consistent. The KCs were then grouped for each of these 1000 $\mathrm{s}$ simulations in the same manner as for the actual data (i.e., within 200 ms time windows) to create the distribution of expected single and multichannel $\mathrm{KC}$ events under the null hypothesis.

Using the same method for subjects with more than two channels, we tested co-occurrence for KCs observed in three or more channels, four or more channels, and five or more channels. Five or more KCs had to be observed for the category to be tested. Bonferroni correction for multiple comparisons was applied to all test results.

To test whether KCs occur in a sequential order across the cortex, a multinomial test was performed in $\mathrm{R}$ for $\mathrm{KC}$ events observed in two, three, four, or five channels. The channel sequence of each KC event was determined by ordering channels by their KC peak latency. For a given set of channels, the multinomial test compares the frequencies of observed sequences to all possible sequences containing the same channels. Bonferroni correction was applied separately to each category of channels over all subjects.
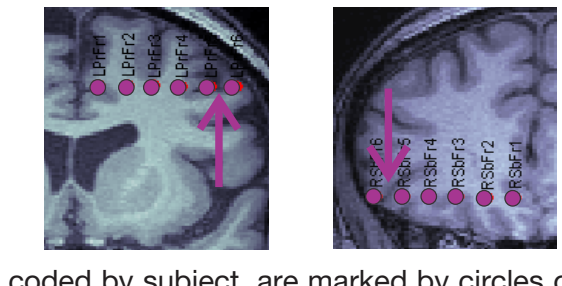
ms window where the template and test data overlap was compared with a predetermined threshold value. If this value was greater, the channel was considered to contain KC-like activity. However, if the time when the sliding inner product was greatest was at the edge of the $200 \mathrm{~ms}$ window (and the amplitude continued to get larger beyond that window), then the activity was not counted as KC like.

The threshold value for KC-like activity was determined for each channel by calculating the sliding inner product between the $\mathrm{KC}$ template and randomly chosen epochs of data that did not contain KCs or slow oscillations in any channel. The number of such epochs chosen was $110 \%$ the number of $\mathrm{KC}$ events for each night. As above, the maximum value of the $200 \mathrm{~ms}$ window where the template and each epoch overlapped was stored in a vector. The threshold was the 99th percentile of that vector. Thus, the KC-like activity chosen by the template can be considered to be more similar than random activity to the template constructed from the manually chosen KCs at $p<$ 0.01 . For 2 nights in two different subjects, it was not possible to obtain $110 \%$ the number of KC events. In these cases, $\sim 350$ periods for each channel were used.

To quantify the characteristic differences between manually detected and template-detected $\mathrm{KCs}$, the $\mathrm{KC}$ peak amplitudes, and the signal variance from -1.4 to $-0.4 \mathrm{~s}$ prior to the KC peak, were calculated for each channel for the null times, manual KCs, and template-detected KCs. The $z$-scores for the peak amplitude and variance on each channel for the manually detected and template-detected $\mathrm{KCs}$ were calculated with respect to the null values. A paired $t$ test was calculated between the $z$-scored manual $\mathrm{KC}$ amplitudes averaged over all channels and the $z$-scored template-detected KC amplitudes averaged over all channels. Another paired $t$ test was calculated comparing the pre-KC period variance between average $z$-scored manually detected and template-detected KCs.

A new list of $\mathrm{KC}$ events, including KC-like activity in addition to the manually marked KCs was computed. To test whether there is a consistent temporal relationship between manually detected and template-detected KCs, the difference between the average peak latency of all manual KCs and the average of all template KCs was calculated for each $\mathrm{KC}$ event for a representative night over all subjects.

The number of channels participating in KCs and the regional distribution of KCs was compared between the manual-only KCs and the manual plus template-detected KCs. For 1 representative night for each subject, the number of KCs occurring on each channel was normalized to the largest number of KCs occurring on a channel. This normalization was performed separately for the manual-only detections and the manual plus detections surviving the 99th percentile threshold. A multinomial test was repeated for these KCs to test whether the inclusion of $\mathrm{KC}$-like activity revealed any meaningful sequential order of two channels across the cortex. Bonferroni correction was applied to all tests.

To further test for a significant relationship between $\mathrm{KC}$ latency and anterior-to-posterior distance in the cortex, all pairs of anterior and posterior channels were chosen for each subject. Pairs were in all cases from different electrodes and could maximally have one contact gap in the medial-lateral direction. These three-dimensional electrode coordinates were projected onto the sagittal plane. The distance between these coordinates for each channel pair was plotted versus the average $\mathrm{KC}$ latency difference between each pair. Additionally, the channel distance was plotted for each individual KC latency difference. Significant distance versus delay relationships were analyzed in a linear mixed-effects model (Pinheiro et al., 2015), where the patient was introduced as a random effect to account for interindividual variability. Marginal $r^{2}$ values were calculated as presented in the study by Nakagawa and Schielzeth (2013).

\section{Results}

\section{Cortical areas generating KCs}

$\mathrm{KCs}$ were analyzed from nine patients undergoing observation for intractable epilepsy. A total of 55 bipolar SEEG contacts were included for analysis. Contacts sampled all lobes but showed the greatest concentration in subcallosal, prefrontal (anterior and posterior), and temporoparieto-occipital junction regions (Fig. 1A). Bipolar contact pairs spanned the gray matter, with one contact in the white matter and the other in the CSF, providing a very focal measure (Fig. 1B).

KCs were manually marked, independently on each bipolar channel. A total of 13,821 KCs were manually marked over all channels for all subjects. For each channel, KCs were verified as down states by a significant drop in high gamma power at the time of the KC (Fig. 2). Locally generated KCs were demonstrated in all sampled regions, including subcallosal, orbital, cingulate (anterior and posterior), frontal (superior, middle and inferior, pars opercularis, triangularis, and orbitalis), supramarginal, angular, annectant, temporal (inferior and middle) gyri, the insula and frontal operculum, and the central and anterior occipital sulci (Fig. 2).

In addition to the 55 bipolar SEEG contacts included for further analysis, KCs were also identified in four bipolar SEEG contacts where it has previously been reported that KCs are not found (Wennberg, 2010). These sites were in anteroventral medial temporal (possibly entorhinal), posteroventral medial temporal, and lateral ventral temporal areas. These four sites were not included in further analyses because they either occurred in potentially nonisocortex (entorhinal cortex) or were in locations implicated in the patient's epilepsy; therefore, further verification is needed for these sites. As with the other pairs, these sites recorded locally generated KCs, as verified by a significant drop in high gamma power (Fig. 2, sites marked by red squares).

\section{KCs measured in bipolar versus common average reference montage}

Previously, KC generation was examined using SEEG, with each individual electrode contact referenced to a common average of scalp electrodes (Wennberg, 2010). To compare this method to the method of transcortical 


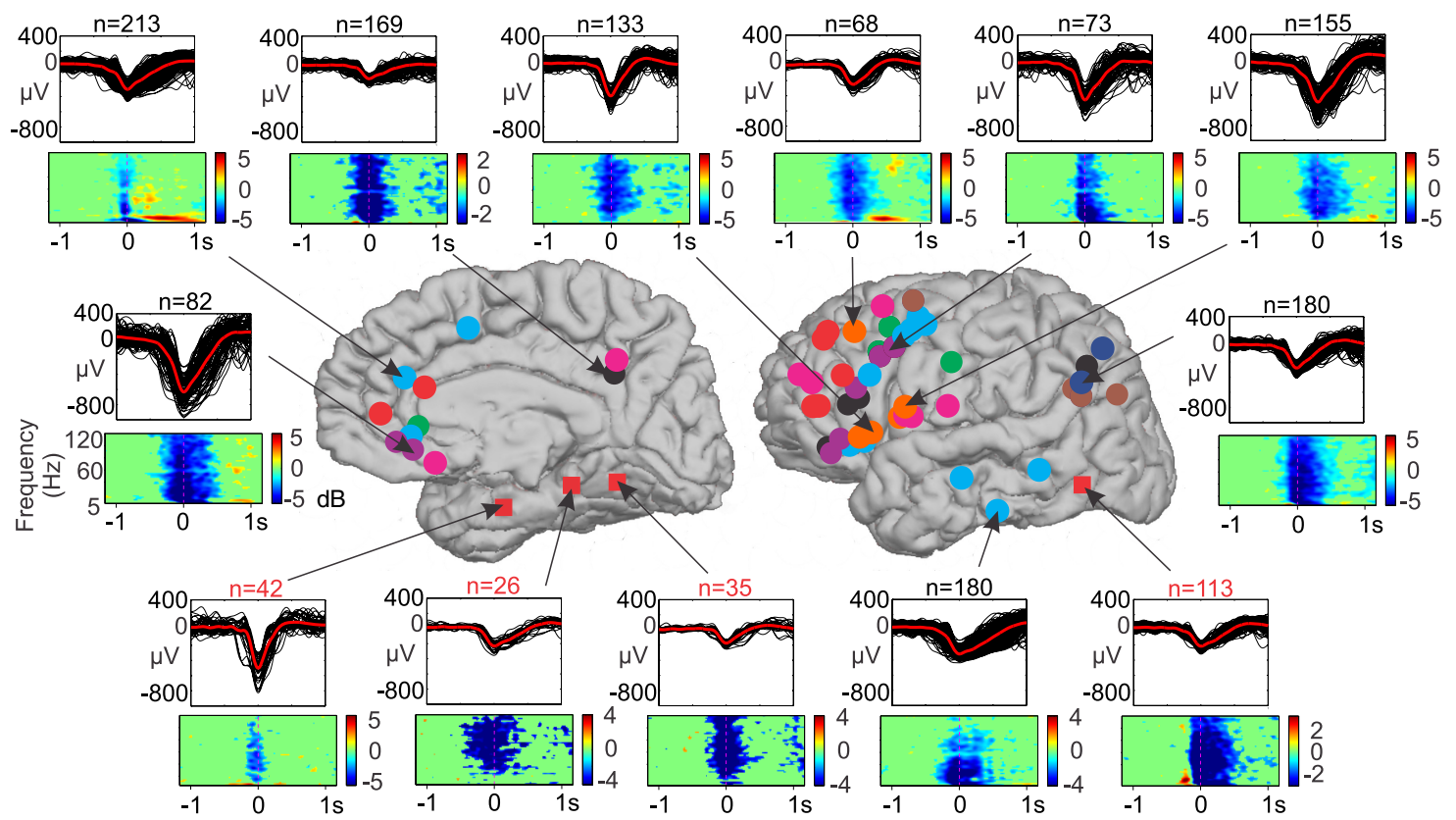

Figure 2. Example KC localizations, waveforms, and down state confirmations. KCs are plotted from representative sites across the cortical areas sampled. Individual KCs for each bipolar location are plotted in black with the average KC overlaid in red. The number of individual KCs plotted is listed above the waveforms. Below the KC waveforms, time frequency plots from 5 to $120 \mathrm{~Hz}$ with a -1.5 to $-0.5 \mathrm{~s}$ baseline relative to the most negative peak of the $\mathrm{KC}$ at time zero indicate a significant drop ( $p<0.01$, uncorrected) in high gamma power at the time of the KC. Arrows indicate the bipolar locations where the plotted waveforms and time-frequency plots were recorded. Circles indicate the subject color-coded bipolar channel locations, as in Figure 1. The four additional red squares indicate sites where KCs were located, but not included for additional analysis because they were part of an epileptic region or potentially in entorhinal cortex.

bipolar subtraction used in the analyses presented here, KCs were examined in two adjacent white matter contacts, as well as two adjacent contacts spanning the gray matter, in both the common average reference and bipolar montage (Fig. 3). All four contacts were located on the same electrode.

Using the KCs identified on the bipolar channel spanning the gray matter, the two white matter contacts referenced to the common scalp average show clear KCs (Fig. 3A, red waveforms). However, when the two white matter channels are viewed in a bipolar montage (blue waveform), the signal is flat. This finding implies that the common average reference signal in this case is due to volume conduction rather than local generation. This interpretation is consistent with the absence of a drop in high gamma power in any bipolar or referential recording from the white matter contacts.

Conversely, the transcortical bipolar contact along the same electrode used in this study spans the gray matter, with one contact on the white matter side of a cortical patch, and the other on the CSF side. In this case, both channels referenced to the common scalp average show $\mathrm{KCs}$ (Fig. 3B, red waveforms), but so does the bipolar subtraction between these two channels (Fig. 3B, blue waveform). Additionally, in all three cases, there is a drop in high gamma power to verify that what is being recorded is a down state. A common average reference SEEG can, therefore, accurately record $\mathrm{KCs}$, but it also risks record- ing activity that is not locally present, which is not the case for bipolar SEEG.

\section{$\mathrm{KC}$ amplitude and occurrence rate varies across cortical locations}

In Figure $4 A$, average $\mathrm{KC}$ amplitudes for each channel are color coded by subject. The mean amplitudes for subjects 1 through 9 were as follows (in $\mu \mathrm{V}$ ): 416, 329, 209, 489, $385,513,420,624$, and 230 . KCs with the largest amplitude were recorded in posterior prefrontal and anterior cingulate/subcallosal areas, whereas small $\mathrm{KCs}$ were recorded in anterior prefrontal and orbital areas, and moderate amplitude KCs were recorded in ventrolateral temporal areas (Figs. 2, 4A). Large KCs were also recorded in supramarginal, posterior cingulate, and anterior occipital areas, although sampling was limited. It should be appreciated that low amplitudes could reflect individual variation between patients, in exact electrode placement with respect to the cortex, or in variable spacing between contacts, rather than a characteristic of a particular cortical area.

In order to account for these patient and channel variabilities, a linear mixed-effects model of the amplitudes was performed with location as a fixed effect, patient as a random effect, and channel as a random nested effect. Bipolar channels were grouped into 10 locations by anatomic location, as pictured in Figure 1. The results of the model revealed that there were no significant amplitude differences between areas, although the anterior prefron- 
A

LPF2-3

LPF2 in white matter LPF3 in white matter Not included in analysis
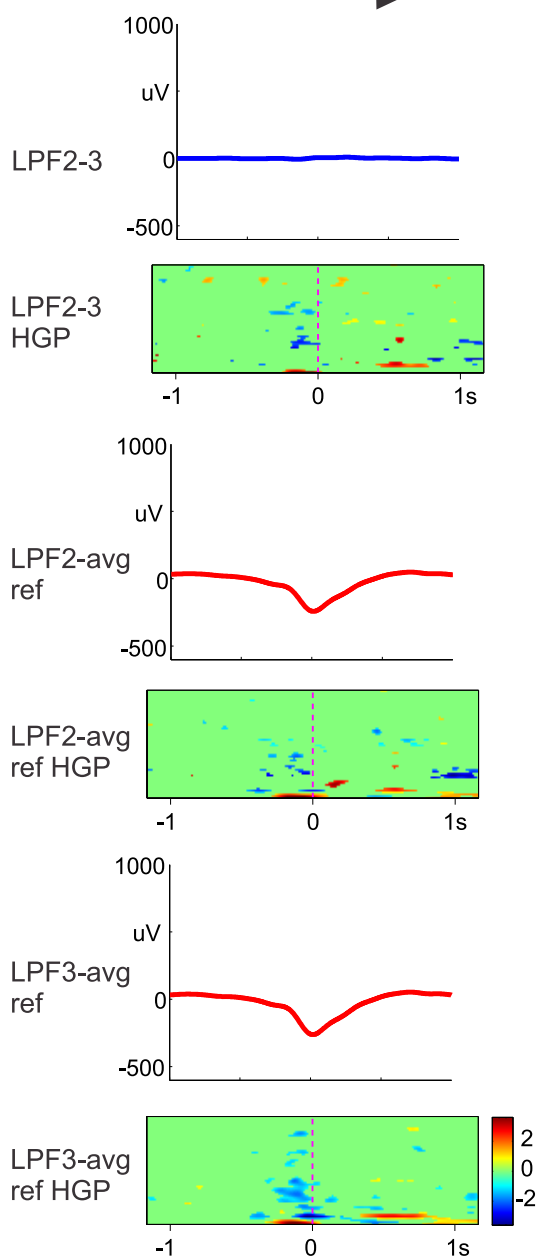

B

LPF5-6

LPF 5 in grey matter

LPF6 in white matter

Included in analysis
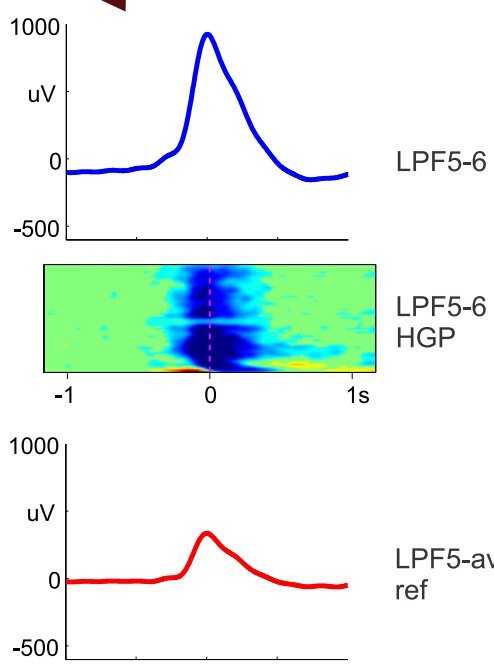

LPF5-avg

ref

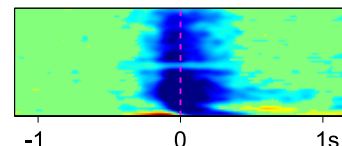

LPF5-avg

ref HGP

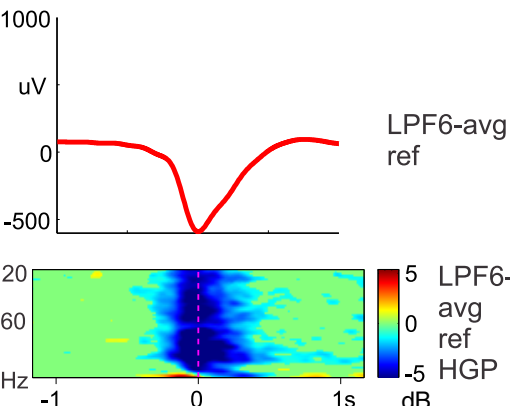

Figure 3. Bipolar versus common average reference recordings. Bipolar $\mathrm{KC}$ recordings (blue waveforms) were compared with monopolar KC recordings (red waveforms) referenced to a common average reference (T3, T5, F3, C3, P3, O1, F4, C4, P4, O2, T4, and T6) as in Wennberg (2010). Two pairs of adjacent contacts located on the same electrode of subject 4 were examined. $\boldsymbol{A}$, Bipolar (blue) and referential (red) recordings from two adjacent contacts, both located in the white matter, as confirmed by MRI. Bipolar recordings show no KCs, but referential recordings do, despite the fact that no decrease in high gamma power is present in the time-frequency plots below each waveform. Thus, referential recordings show KCs even when they are not locally generated. $\boldsymbol{B}$, Bipolar (blue) and referential (red) recordings from two adjacent contacts that span the gray matter, as confirmed by MRI. Both bipolar and referential recordings show KCs, together with decreased high gamma power. All recordings were averaged with respect to the peaks of KCs identified in the bipolar recordings in $\boldsymbol{B}$.

tal cortex was close to being significantly smaller $(p=$ 0.089), despite the common observation that the largest scalp KCs are recorded above this area. These issues are further examined below.

In Figure $5 A$, the occurrence rate in each bipolar pair was normalized to the pair with the highest frequency in that subject, resulting in less striking differences between cortical locations. As a measure of occurrence rate, the
inter-KC intervals were calculated for each channel. A linear mixed-effects model of these intervals was calculated with location as a fixed effect and patient as a random effect to test for areas with significantly different occurrence rates. Compared with the orbitofrontal reference, KCs occurred $15 \%$ more often in the anterior cingulate $(p=0.0051), 48 \%$ more often in posterior cingulate $(p<0.00001), 11 \%$ more often in posterior prefrontal 


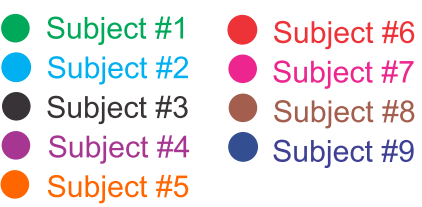

A Manual
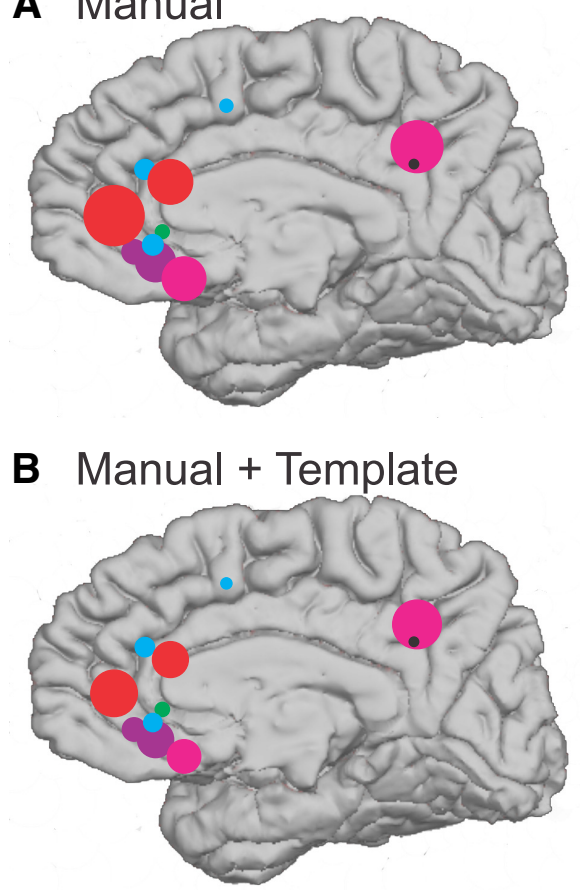

Amplitude:

- $250 \mu \mathrm{V}$

$500 \mu \mathrm{V}$

$750 \mu \mathrm{V}$
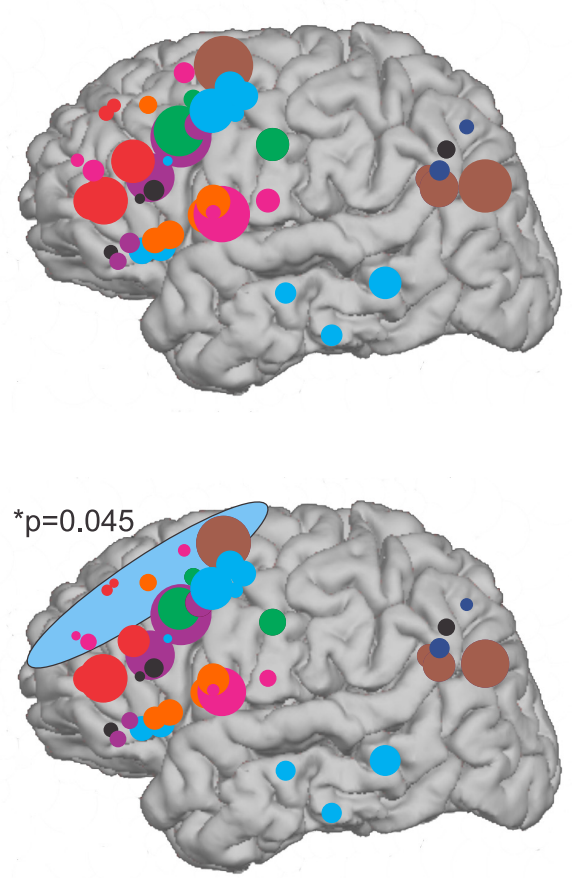

Figure 4. KC amplitudes vary across the cortex. $\boldsymbol{A}$, The average amplitude of manual KCs at each channel, color coded by subject, is highly variable across the cortex. However, no significant effects are found when a linear mixed-effects model is applied. $\boldsymbol{B}$, The addition of KC-like activity shows a similar pattern, but with the increased sample, the same linear mixed-effects model now finds that the anterior prefrontal channels highlighted by the blue circle are $\sim 30 \%$ smaller than the orbitofrontal reference $(p=0.045)$. Black circles in the top right indicate the amplitude scale represented by the size of each circle.

cortex $(p=0.024)$, and $14 \%$ more often in ventrolateral temporal regions $(p=0.022)$; these groups are highlighted in pink in Figure $5 \mathrm{~A}$. Again, contrary to inferences from scalp EEG findings, KCs occurred $30 \%$ less often in the anterior prefrontal cortex $(p=0.0001)$ compared with the orbitofrontal location. KCs also occurred two times less frequently in the central sulcus $(p<0.00001)$; these two locations are highlighted in blue in Figure $5 A$.

\section{$\mathrm{KCs}$ co-occur in channels across the cortex}

In order to determine whether KCs co-occur across the cortex, the KCs that were marked independently on each channel were regrouped. Starting with the first KC latency, any KCs occurring in other channels within the next $200 \mathrm{~ms}$ were grouped as being part of the same KC event. As the time delay of potential KC propagation across the cortex is unknown, this 200 ms time window was defined for grouping a KC event because it is the approximate time delay reported for the slow oscillation from anterior to posterior scalp EEG recording sites (Massimini et al., 2004). Each KC event, therefore, would minimally contain one channel and could maximally contain the number of channels analyzed for an individual subject.
To examine whether KC peaks in different channels participating in a KC spanned the entire $200 \mathrm{~ms}$ window, the time between the first and last peak in each $\mathrm{KC}$ containing more than one channel was calculated. The mean and SD of these maximum peak delays was calculated for each night for each subject. All subjects showed a mean peak difference of $<100 \mathrm{~ms}$ (Table 2). The single exception was 1 night for subject 9, where only one KC involved both channels; as a result, the mean reflects only one calculation. Therefore, even though a $200 \mathrm{~ms}$ window was chosen to include KCs potentially traveling across the cortex, KCs occurred in almost all cases in less than half this time.

To test whether these observed KC co-occurrences were significant, we simulated a set of expected $\mathrm{KC}$ data under the null hypothesis that the $\mathrm{KC}$ times are unrelated in different channels. This expected distribution was created by randomizing the inter-KC intervals within channels of the observed KCs and grouping the latencies over channels within $200 \mathrm{~ms}$ windows, as was done with the observed KCs. We examined observed and expected $\mathrm{KCs}$ occurring in one channel versus two or more channels for each subject (Fig. 6). In the observed KCs, there 


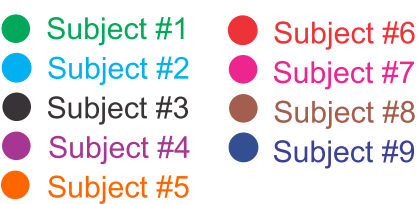

Normalized Occurrence Rate:

- $25 \%$

- $50 \%$

$100 \%$

\section{A Manual}
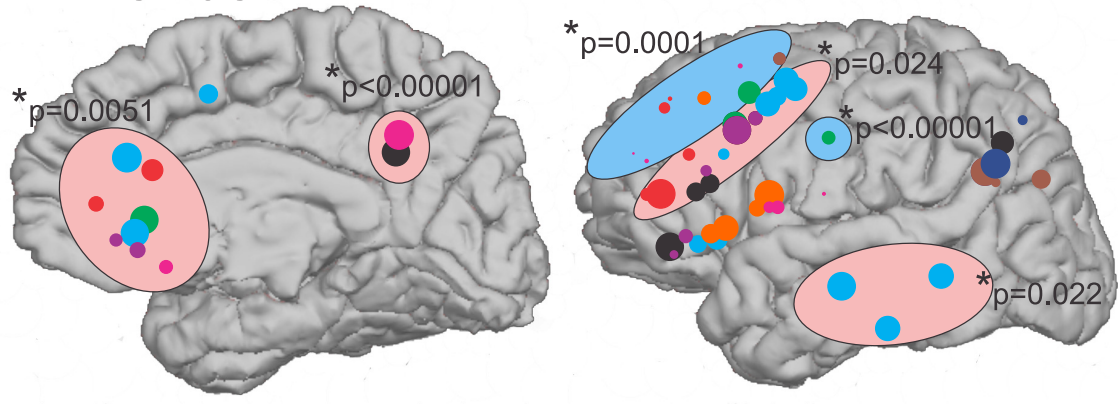

B Manual + Template
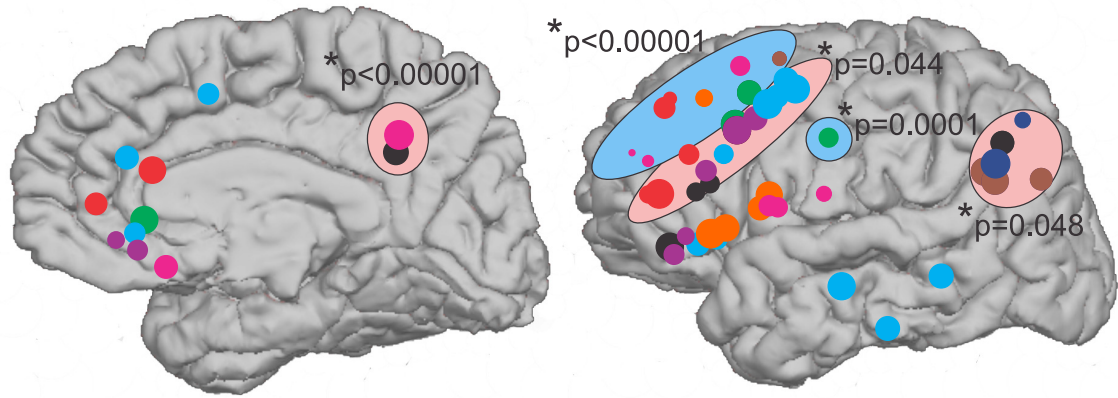

Figure 5. KC occurrence rates vary across the cortex. $\boldsymbol{A}$, Manually marked KCs color coded for each subject. The size of the circle indicates the $\mathrm{KC}$ occurrence rate at each location, normalized to the maximum $\mathrm{KC}$ occurrence rate seen on any channel for that subject. Black circles in the top right indicate this normalized occurrence rate scale. Locations identified by a linear mixed-effects model to have a significantly higher occurrence rate than the orbitofrontal reference are highlighted by pink circles. Locations identified as occurring significantly less frequently are highlighted by blue circles. $\boldsymbol{B}, \mathrm{KC}$-like activity in addition to manually marked KCs. The locations showing a lower occurrence rate are the same as in $\boldsymbol{A}$. The locations showing a higher occurrence rate differ with the addition of the template-detected KCs.

are KCs that occur only in one channel (Fig. 6A, light orange). However, there are many more KCs that occur only in one channel for the null distribution (Fig. 6B, light purple). Conversely, there is a larger number of KCs involving two or more channels for the observed data (Fig. $6 A$, dark orange) than under the null hypothesis (Fig. $6 B$, dark purple). To statistically verify this observation, a Fisher's exact test was performed, comparing the number of observed $\mathrm{KCs}$ to the null distribution in one channel versus two or more channels. For 20 nights over eight subjects, this was highly significant $(p<0.05$, Bonferroni corrected; Table 3 ). For 4 nights over two subjects (3 of 4 nights for one subject and 1 of 3 nights for the other subject), it was not. Due to the variable number of channels for each subject, the "one channel" versus "two or more channels" comparison was the only comparison testable for all nine subjects. In eight subjects, it was possible to repeat this test using expected and observed data for one channel and three or more channels; for seven of these subjects at least 1 night was significant at $p<0.05$ (Bonferroni corrected), while the night of the eighth subject was not significant. For six subjects, it was possible to test up to four or more channels; in this case, we find all nights for five subjects to be significant at $p<$ 0.05 (Bonferroni corrected) and not in 1 night in one subject. This analysis demonstrates that KCs co-occur across the cortex.

\section{KCs do not occur in particular sequential order across the cortex}

As examined above, channels participating in a $\mathrm{KC}$ event exhibit a tighter time window than the $200 \mathrm{~ms}$ used to group KC events. However, this specific limit was imposed to investigate propagation sequences: according to Massimini et al. (2004), it takes $\sim 200 \mathrm{~ms}$ for a slow wave to travel from anterior to posterior cortex. As stated previously, because the potential propagation time of KCs is unknown, the time delay of the SO was used as a 
Table 2. Mean and SD of maximum KC peak differences between manually chosen $\mathrm{KCs}$ for each night for each subject

\begin{tabular}{|c|c|c|c|}
\hline Subject & Channels $(n)$ & $\begin{array}{l}\text { Mean of maximum KC } \\
\text { peak differences (ms) }\end{array}$ & $\mathrm{SD}(\mathrm{ms})$ \\
\hline 1 & 4 & 61.1 & 49.5 \\
\hline 2 & 13 & 99 & 59.6 \\
\hline 3 & 5 & 86.3 & 59.5 \\
\hline 4 & 7 & 66.3 & 54.1 \\
\hline \multicolumn{4}{|l|}{5} \\
\hline N1 & 5 & 24.8 & 23.8 \\
\hline N2 & 5 & 27.8 & 26.4 \\
\hline N3 & 5 & 32.2 & 27.7 \\
\hline \multicolumn{4}{|l|}{6} \\
\hline N1 & 7 & 71.3 & 48.5 \\
\hline N2 & 7 & 66.4 & 45.6 \\
\hline N3 & 7 & 81.2 & 52.8 \\
\hline N4 & 7 & 60.3 & 54 \\
\hline \multicolumn{4}{|l|}{7} \\
\hline N1 & 8 & 68.6 & 59.1 \\
\hline N2 & 8 & 63.2 & 57.2 \\
\hline N3 & 8 & 73.4 & 61.2 \\
\hline N4 & 8 & 77.7 & 63.1 \\
\hline N5 & 8 & 70.7 & 51.1 \\
\hline N6 & 8 & 74.9 & 59.7 \\
\hline \multicolumn{4}{|l|}{8} \\
\hline N1 & 4 & 49.8 & 53.4 \\
\hline $\mathrm{N} 2$ & 4 & 79.2 & 42 \\
\hline N3 & 4 & 67.5 & 59.7 \\
\hline \multicolumn{4}{|l|}{9} \\
\hline N1 & 2 & 95.2 & 59.4 \\
\hline N2 & 2 & 115.2 & 0 \\
\hline N3 & 2 & 52.2 & 39 \\
\hline N4 & 2 & 78.6 & 83 \\
\hline
\end{tabular}

maximum time window. To test the hypothesis that KCs occur in a sequential order across the cortex, we performed a multinomial test on the sequential order of channels participating in the observed KCs. Using the KC peak latencies, we recorded for a pair of channels the number of times the KC peak occurred in channel A before channel $B$ and the number of times the KC peak occurred in channel $B$ before channel $A$. In this example of two channels, the multinomial test determined when one sequential order occurred significantly more often than the other sequential order. Of 341 channel pairs tested over a representative night for each of the nine subjects, only 20 pairs from two subjects (subjects 2 and 6) were statistically significant at $p<0.05$ after Bonferroni correction. These results indicate that when two channels repeatedly record KCs within a 200 ms time window, only $6 \%$ of these 341 channel pairs show a significantly consistent latency order. Conversely, $94 \%$ of channel pairs show no systematic latency even though they occur repeatedly within the same transcortical KCs.

If KCs significantly occurred in a sequential order from anterior to posterior cortex, we would expect to find ordered sequences of two or more channels, beginning in anterior cortical regions and finishing in posterior cortical regions. Thus, we examined individually all of the electrode pairs to determine whether they were systematically engaged in an anterior-to-posterior direction. We found that for subject 2 ,
11 of the 16 significant pairs started in various parts of cortex and ended in the temporal lobe. Three other pairs were situated along the same electrode, one was a pair of mirrored locations across the hemispheres, and the last was a pair with a slight posterior-to-anterior direction (Fig. 7B, red arrows). Of the four significant pairs found for subject 6 , there was evidence of anterior-to-posterior propagation for three pairs, all lying entirely within prefrontal cortex. One of these three pairs was along the cingulate gyrus. One other pair was located along the same electrode, with no anteriorposterior separation.

We further tested three-, four-, and five-channel sequences, whenever possible given the number of channels and the rate of multichannel $\mathrm{KC}$ occurrence for each subject. Of 222 triplets tested, 5 from subject 6 were statistically significant at $p<0.05$ after Bonferroni correction. As with the pair results for this subject, these triplets showed anterior-to-posterior propagation only within the prefrontal cortex, as well as patterns that bounced back and forth or looped around within the frontal cortex. There were no significant sequences involving four or five channels.

\section{Automatic template-based detection of KC-like activity}

The above analyses were performed on the manually marked KCs. The strict criteria used to select these phenomena, most notably that there was a flat baseline (i.e., no preceding oscillation) prior to the $\mathrm{KC}$, may have prevented $\mathrm{KC}$-like phenomena from being detected, and thus result in an underestimation of the degree of $\mathrm{KC}$ cooccurrence. In order to detect KC-like events, a template was created for each channel and applied at the times of manual KCs occurring in at least one channel (Fig. 8). A threshold was established as the 99th percentile of periods when no KC was visually present. Events that exceeded the template threshold were included as KC-like events (see Materials and Methods). Application of these individualized channel templates identified 13,383 KC-like events-a 96.8\% increase in detected KCs-and $98.7 \%$ of the 13,821 manually marked KCs.

Compared to the manually detected $\mathrm{KCs}$, templatedetected KCs were smaller in amplitude (manual $=420 \pm$ $219 \mu \mathrm{V}$ vs template $=326 \pm 183 \mu \mathrm{V}$ ) and had more variance in the pre-KC period (manual KCs $=1945 \pm 1604$ vs template-detected $\mathrm{KCs}=7388 \pm 10,708)$. The variance in the preactivity period (Fig. $9 A$ ) and the KC peak amplitude (Fig. 9B) for each of subject 1's four channels are plotted for the null times (Fig. 9B, black distribution), manual KCs (Fig. $9 B$, red distribution), and template-detected KCs (Fig. 9B, blue distribution). In some cases, the template-detected $\mathrm{KCs}$ were smaller in amplitude than the manual KCs, without much distinction in the variance (channel 1), while in other cases, the opposite occurred: the variance was larger for template-detected KCs than for manual KCs, without a large distinction in amplitude (channel 4). In most cases, it was a combination of variance and amplitude that distinguishes the manually detected and template-detected KCs (channels 2 and 3 ). 
A
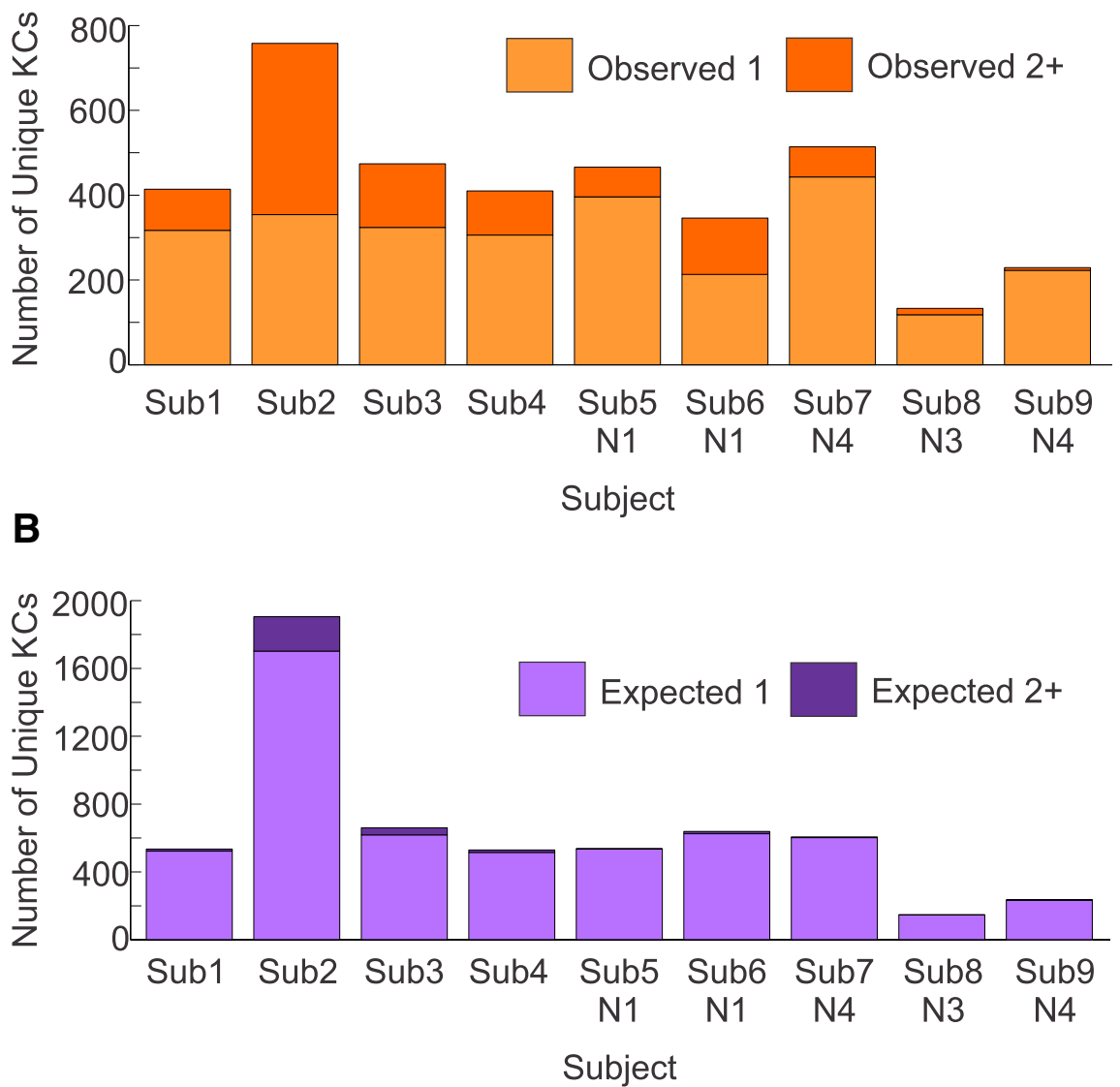

Figure 6. Observed manual $\mathrm{KCs}$ versus those expected under the null hypothesis of independent occurrence, for representative nights for each subject. $\boldsymbol{A}, \mathrm{KC}$ events observed for each subject were classified into two groups: KCs with one participating channel (light orange); and KCs with two or more participating channels (dark orange). $\boldsymbol{B}, \mathrm{KC}$ events derived from simulating an expected distribution for each subject were classified into the same two groups: one channel (light purple); and two or more channels (dark purple). For all subjects, there are more KCs that occur only in one channel expected under the null hypothesis than were actually observed. These distributions are tested statistically using a Fisher's exact test, and these results are listed in Table 3.

In order to evaluate for all channels the contributions of $\mathrm{KC}$ peak amplitude and pre-KC period variance in distinguishing manual from template-detected KCs, $z$-scores were calculated for the KC peak amplitude and pre-KC period variance for each channel with respect to the null values (Fig. $9 \mathrm{C}$, right, which is an expansion of the lower left corner outlined in gray). In general, as above, the manual KCs (Fig. 9C, red circles) are larger in amplitude (i.e. shifted to the right on the $x$-axis) than the template-detected KCs (Fig. 9C, blue triangles). The manually detected and templatedetected values for each channel are connected by a line. This line is green if the manual KCs were larger in amplitude than the template-detected KCs; however, this line is black in the rare cases where the templatedetected KCs are larger in amplitude than the manual $\mathrm{KCs}$. Furthermore, the pre-KC period variance is generally larger (i.e. shifted up on the $y$-axis) for the template-detected KCs compared to the manual KCs within each channel. To test these observations, a paired $t$ test between the manually detected and template-detected KC $z$-transformed amplitudes over all channels was significant $(p=0.000065)$, as was a paired $t$ test between the $z$-transformed variance in the pre-KC period for manually detected and templatedetected KCs over all channels $(p=0.00027)$. While there are clear overall distinctions in amplitude and pre-KC period variance between template-detected and manually marked KCs, they appear to form a continuum on a channel-by-channel basis, and thus both manually detected and template-detected KCs should be considered together when examining the true extent of cortical involvement at the time of KCs.

We further examined whether a directionally consistent temporal lag existed between manually detected and template-detected KCs by subtracting the average of the template $\mathrm{KC}$ peaks from the average of the manual $\mathrm{KC}$ peaks within each $\mathrm{KC}$ event. The mean lag was $-1.25 \mathrm{~ms}$ with an SD of $40 \mathrm{~ms}$, and the normality of the distribution was determined visually; therefore, we concluded that no such temporal relationship existed between the manually detected and template-detected KCs. 
Table 3. Fisher's exact test for co-occurrence for one channel and two or more channels; three or more channels; four or more channels; and five or more channels for all nights for all subjects

\begin{tabular}{|c|c|c|c|c|}
\hline $\begin{array}{l}\text { Subject } \\
1\end{array}$ & $\begin{array}{l}1 \text { Channel and } \geq 2 \text { channels } \\
<2.2 E-16\end{array}$ & $\begin{array}{l}\text { 1 Channel and } \geq 3 \text { channels } \\
9.49 \mathrm{E}-12\end{array}$ & $\begin{array}{l}\text { 1 Channel and } \geq 4 \text { channels } \\
0.000445\end{array}$ & 1 Channel and $\geq 5$ channels \\
\hline 2 & $<2.2 \mathrm{E}-16$ & $<2.2 \mathrm{E}-16$ & $<2.2 E-16$ & $<2.2 \mathrm{E}-16$ \\
\hline 3 & $<2.2 \mathrm{E}-16$ & $<2.2 \mathrm{E}-16$ & 6.24E-09 & 0.00493 \\
\hline 4 & $<2.2 \mathrm{E}-16$ & $<2.2 \mathrm{E}-16$ & $5.38 \mathrm{E}-08$ & \\
\hline \multicolumn{5}{|c|}{ 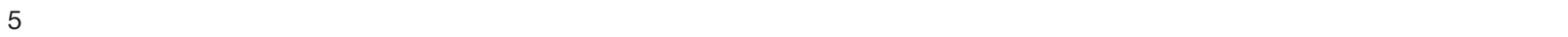 } \\
\hline N1 & $<2.2 \mathrm{E}-16$ & & & \\
\hline N2 & $5.40 E-14$ & & & \\
\hline N3 & $2.96 \mathrm{E}-13$ & 0.0135 & & \\
\hline \multicolumn{5}{|c|}{ - } \\
\hline N1 & $<2.2 \mathrm{E}-16$ & $<2.2 \mathrm{E}-16$ & $<2.2 \mathrm{E}-16$ & $1.10 E-15$ \\
\hline N2 & $<2.2 \mathrm{E}-16$ & $<2.2 \mathrm{E}-16$ & $<2.2 \mathrm{E}-16$ & $<2.2 \mathrm{E}-16$ \\
\hline N3 & $<2.2 \mathrm{E}-16$ & $<2.2 \mathrm{E}-16$ & $<2.2 \mathrm{E}-16$ & $1.25 E-15$ \\
\hline N4 & 3.92E-10 & 0.0125 & & \\
\hline \multicolumn{5}{|c|}{ (4) } \\
\hline N1 & $<2.2 \mathrm{E}-16$ & 2.33E-06 & & \\
\hline N2 & $<2.2 \mathrm{E}-16$ & 1.20E-05 & & \\
\hline N3 & $<2.2 \mathrm{E}-16$ & 1.61E-05 & 0.0140 & \\
\hline N4 & $<2.2 \mathrm{E}-16$ & 2.00E-08 & & \\
\hline N5 & 9.50E-06 & & & \\
\hline N6 & 8.09E-08 & & & \\
\hline \multicolumn{5}{|c|}{ 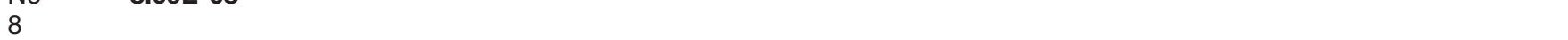 } \\
\hline N1 & $<2.2 \mathrm{E}-16$ & 0.000355 & & \\
\hline N2 & 0.00227 & & & \\
\hline N3 & 0.000107 & & & \\
\hline \multicolumn{5}{|c|}{ ית } \\
\hline N1 & 0.215 & & & \\
\hline N2 & 0.497 & & & \\
\hline N3 & 0.000312 & & & \\
\hline N4 & 0.0140 & & & \\
\hline
\end{tabular}

Bonferroni correction at $p<0.05$ was applied across all tests. Values in bold are significant after this Bonferroni correction.

\section{Including KC-like activity increases the multichannel participation of KCs}

Combining the number of manually marked KCs with the template KCs resulted in 27,204 total KCs over all subjects. We first examined how the addition of template KCs increased multichannel participation. In subjects where all channels participated in a subset of manually marked $\mathrm{KCs}$, the percentage of such KCs increased with the addition of template-detected KCs (Fig. 10A). Similarly, in subjects where only a subset of channels participated in manually marked $\mathrm{KCs}$, the addition of template KCs resulted in KCs where all possible channels participated (Fig. 10B). Manually marked KCs already showed significant co-occurrence across the cortex; the addition of $\mathrm{KC}$-like activity indicates that KCs co-occur even more broadly across the cortex.

In Subjects 1, 2, 3, and 9, all channels participated in a subset of manually marked KCs. For subject 1 , the percentage of KCs involving all four channels increased from about $2 \%$ in manual $\mathrm{KCs}$ to $9 \%$ after addition of the template KCs. For subject 2 (Fig. 10A), all 13 channels participated in only $0.7 \%$ of manual KCs, which increased to $\sim 5 \%$ of all KCs with the addition of KC-like activity. If $\geq 10$ channels are considered as a group for subject 2 , then the percentage of total KCs increased from $\sim 4 \%$ in the manual case to $20 \%$ in the case of manual plus template-detected KCs. For subject 3 , the increase in $\mathrm{KCs}$ involving all channels was slight, from $1 \%$ to $2.5 \%$, while for subject 9 this increase was from $\sim 3 \%$ to $\sim 28 \%$. While this latter result indicates the largest increase, there are only two channels for subject 9 .

In subjects 4, 5, 6, 7, and 8, KCs with all channels participating were not found until template-detected KCs were included. In subject 4 , only five of seven channels maximally participate in manually marked $\mathrm{KCs}$, and these represent only $0.2 \%$ of $\mathrm{KCs}$. With the addition of template-detected KCs, KCs may now involve five, six, or seven channels, accounting for $>12 \%$ of all of the KCs for subject 4 . For subject 5 , manual $\mathrm{KCs}$ only maximally involve three of five possible channels, representing $0.6 \%$ of KCs. After template detections, $>38 \%$ of the subject's KCs involve three, four, or five channels. For subject 6 (Fig. 10B), only $2 \%$ of manually marked KCs involve six of seven channels. After template application, this increases to almost $20 \%$, and the number of KCs involving all seven channels is just under $11 \%$. In subject 7 , a maximum of only five of eight possible channels participate in manual $\mathrm{KCs}$, and $86 \%$ of KCs only involve one channel. After template detection, KCs involving six, seven, and eight channels collectively account for $\sim 7 \%$ of KCs. For subject 8 , only three of four channels maximally participate in the manual KCs, but after including KC-like activity, 6\% of KCs include all four channels.

A 200 ms detection window on manually marked KCs was chosen based on the differences in KC peak latencies reported at the scalp for SOs (Massimini et al., 2004). 


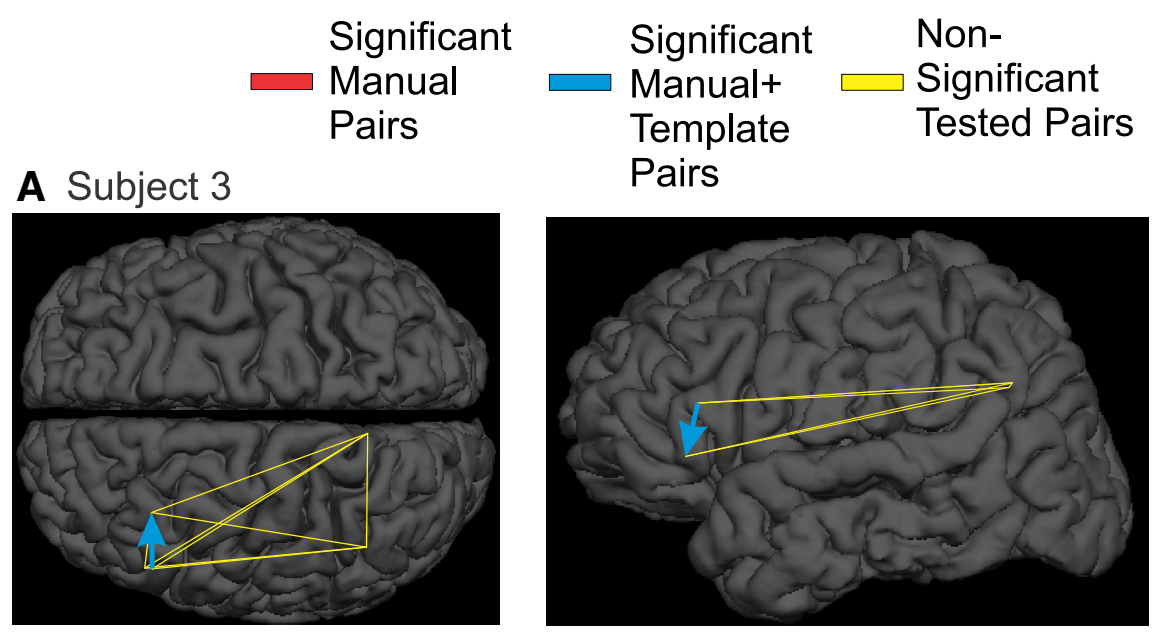

\section{B Subject 2}
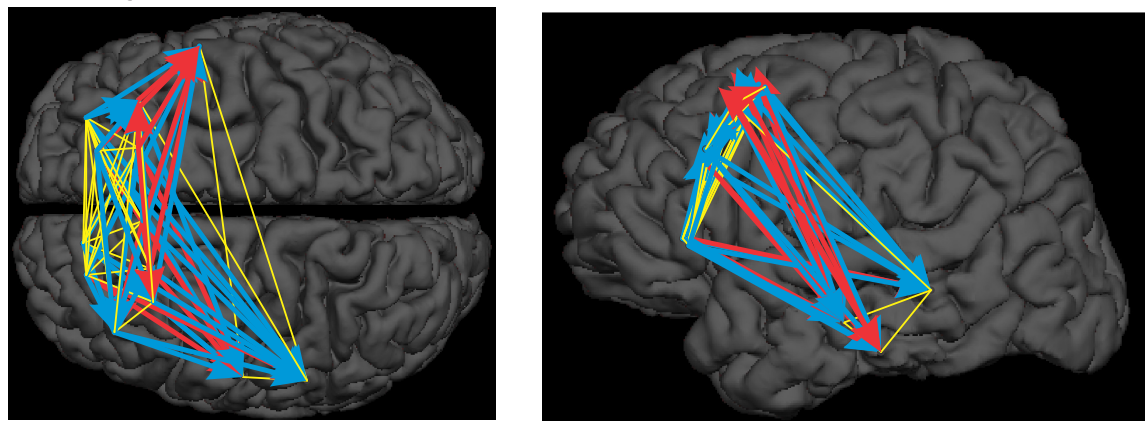

Figure 7. Lack of systematic sequential order in KCs. Sequence pairs that are significant with manually marked KCs are plotted with red arrows, while sequence pairs that are significant only after the addition of KC-like activity are plotted with blue arrows. Sequence pairs tested but not significant are plotted in yellow. $\boldsymbol{A}$, Subject 3 has electrodes in frontal and parietal cortices, but only shows significant sequences within the frontal lobe, and only after the addition of KC-like activity. $\boldsymbol{B}$, Subject 2 shows a large number of significant sequence pairs (16) in manually marked KCs, the majority of which lead to the temporal lobe (11). This pattern is strengthened with the addition of KC-like activity (18 of 29 additional significant pairs). A variety of other significant sequences are seen within the frontal lobe.

However, if this window was artificially small, then we may have underestimated the true extent of KC co-occurrence. In order to test for this possibility, we recalculated co-occurrence after grouping KCs using a 200 ms "crawl" window (Fig. 10). In the analyses described above, we start at the first KC peak and search within the following $200 \mathrm{~ms}$ window for any other manually marked $\mathrm{KC}$ peaks in other channels. In the crawl analysis, we follow the same procedure, but shift the $200 \mathrm{~ms}$ window to follow the second KC peak. The window continues to shift until there are no further detections within the $200 \mathrm{~ms}$ window. The recalculated co-occurrence histograms showed minimal changes using this procedure. Only three of the nine subjects (subjects 2, 3, and 6) showed an increase of more than one KC with the participation of all channels. Even in these subjects, the effects were minimal, as seen by comparing the red and gray bars in Figure 10. In contrast, adding template-detected KCs had a substantial effect on co-occurrence, as seen by comparing the red and blue bars in Figure 10.

In summary, including only the manually marked KCs, most KCs ( $76 \%$, averaged across all subjects) occurred in a single location, and rarely (1\%) in all locations. However, if template-detected KCs were included, only $15 \%$ oc- curred in a single location, and $27 \%$ occurred in all locations.

\section{After including KC-like activity, KCs are smallest in anterior prefrontal regions}

In Figure 4B, average KC amplitudes, including both manually detected and template-detected KCs, are shown for each channel (color coded by subject). For comparison, the same amplitude data for manual KCs only are plotted in Figure $4 A$. The addition of the template KCs generally decreases the average $\mathrm{KC}$ amplitude at each channel, as seen by a reduction in circle size between Figure $4 A$ and Figure $4 B$, and as shown to be significantly different in Figure $9 C$.

In order to compare the amplitude of KCs in different areas, a linear mixed-effects model of the amplitudes was again performed. The anterior prefrontal channels were found to be $\sim 30 \%$ smaller than the orbitofrontal reference channels $(p=0.045$; Fig. $4 B$, blue circle). Again, this finding is in contrast to what would be predicted by scalp EEG. 


\section{Template: Individual KCs: $\quad \begin{gathered}\text { Manual } \\ \text { Above Threshold }\end{gathered}$}

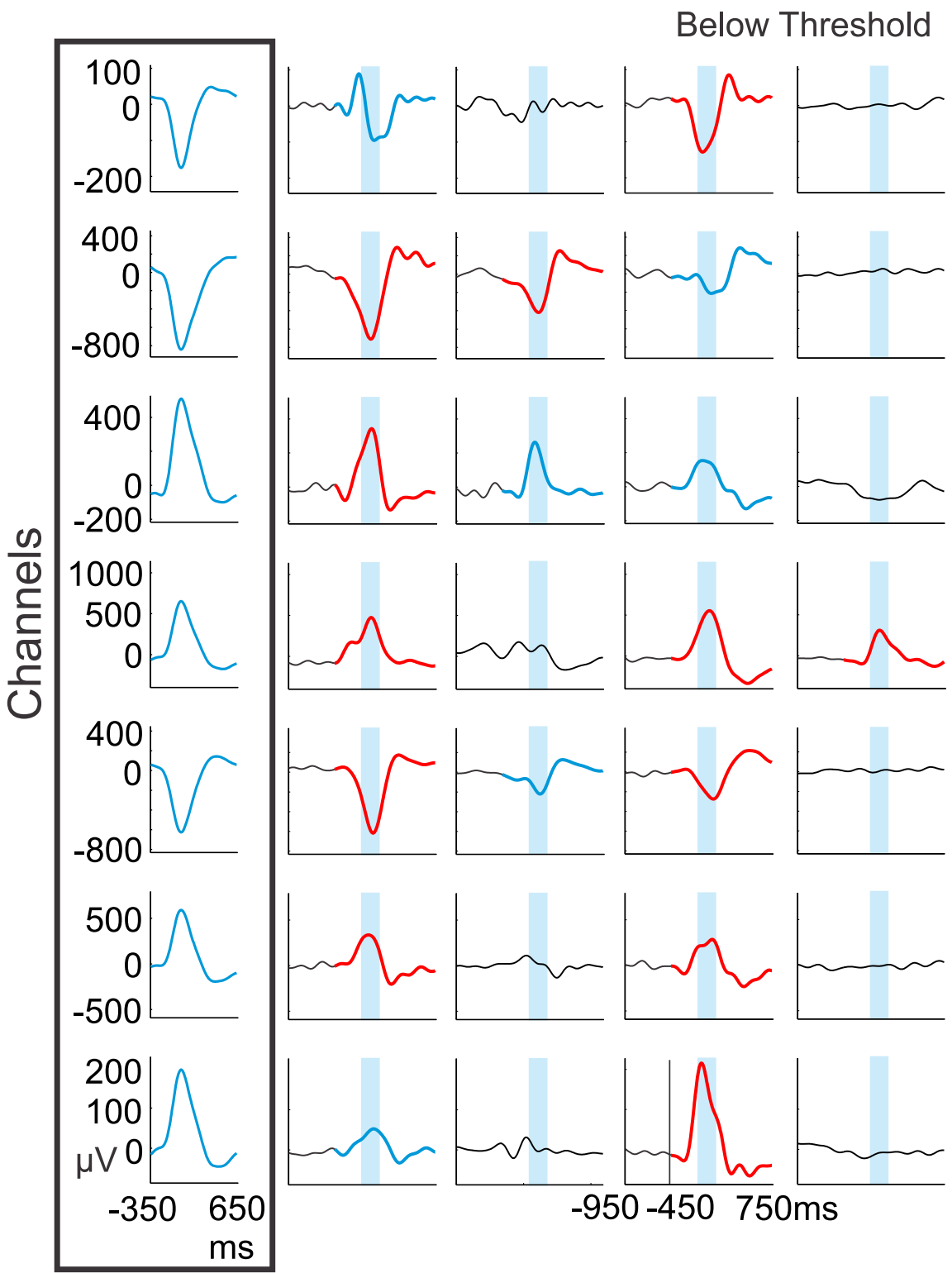

Figure 8. Template application to detect KC-like activity. Channel-specific templates were created for each subject by averaging from -350 to $+650 \mathrm{~ms}$ on the most negative peak at time zero over the manually detected KCs in each channel (box at left). The channel-specific templates were then applied when a manually marked $\mathrm{KC}$ occurred in at least one channel. The templates were applied from -450 to $+750 \mathrm{~ms}$, with time zero representing the average time between the first and last manually chosen channel peaks within a KC. Four such examples from subject 6 are plotted vertically. Manually marked KCs are plotted in red. The maximum value of the sliding inner product between the signal and the template was taken over the blue highlighted window. If the value was above the 99th percentile of the null distribution for that channel and corresponded to the largest (or smallest) peak over the entire $1200 \mathrm{~ms}$ window, the KC was recorded as KC-like activity (KCs in blue), and if it was below the threshold, it was not (signal in black).

\section{Adding KC-like activity has minor effects on the pattern of $\mathrm{KC}$ occurrence rates across cortical locations}

We next examined how the addition of KC-like activity influences the spatial distribution of $\mathrm{KC}$ occurrence rates across the cortex. Again, in Figure 5B, the average KC occurrence rate for each channel is color coded by subject, including both manually detected and template- detected KCs, for comparison with the same occurrence rate data plotted for manual KCs only in Figure $5 \mathrm{~A}$. For each subject, the occurrence rate of KCs in each channel is normalized to the channel with the highest $\mathrm{KC}$ occurrence rate.

Similarly, in order to test whether the KC occurrence rate varies across locations after including templatedetected KCs, a linear mixed-effects model was again 
A

B
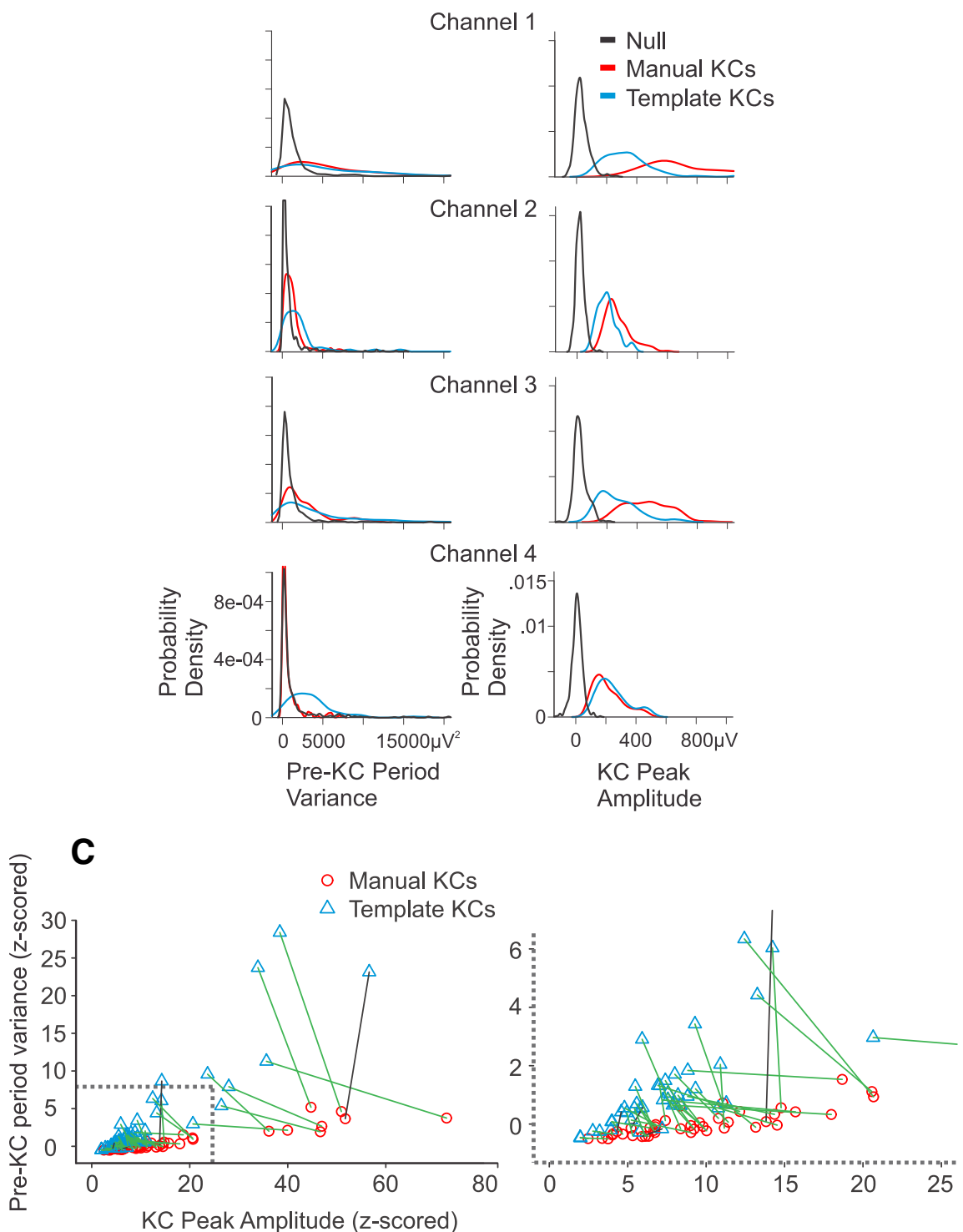

Figure 9. Manually detected KCs have smaller pre-KC period variance and larger amplitude than template-detected KCs. $\boldsymbol{A}$, The probability density of variance in the pre-KC period $(-1.4$ to $-0.4 \mathrm{~s}$ prior to peak) is plotted for the null times (black), manually detected KCs (red), and template-detected KCs (blue) for each of subject 1's four channels. $\boldsymbol{B}$, The KC peak amplitude probability density of null times (black), manually detected KCs (red), and template-detected KCs (blue) are plotted for the same four channels as in $\boldsymbol{A}$. C, For each channel, the manually detected (red circles) and template-detected (blue triangles) z-scores were calculated for the KC peak amplitude and the pre-KC period variance referenced to the null values. A line connects the manually detected and template-detected values for each channel. A green line indicates that the amplitude of template-detected KCs is smaller than that of manually detected KCs in that channel; a black line indicates the opposite relationship. On the right is an expansion of the lower left corner, which is outlined by a gray box.

applied to the inter-KC intervals for each channel. Compared to the orbitofrontal reference, KCs still occur significantly more frequently in posterior cingulate $(26 \%$ more; $p<0.00001)$ and posterior prefrontal $(6 \%$ more; $p$ $=0.044)$. They no longer occur more frequently in anterior cingulate or ventrolateral temporal cortex, but KCs now occur more frequently at the temporo-parieto-occipital junction (15\% more; $p=0.048$ ). These groups are highlighted in pink in Figure $5 B$. As was the case with the manual KCs, the anterior prefrontal KCs occur $26 \%$ less frequently $(p<0.00001)$ and the central sulcus KCs occur $48 \%$ less frequently $(p=0.0001)$ than those in the orbitofrontal channels and are highlighted in blue in Figure $5 B$.

\section{Adding KC-like activity does not reveal any systematic sequential order across the cortex}

We tested whether using only manually marked KCs undersampled $\mathrm{KC}$ activity and therefore, resulted in the 

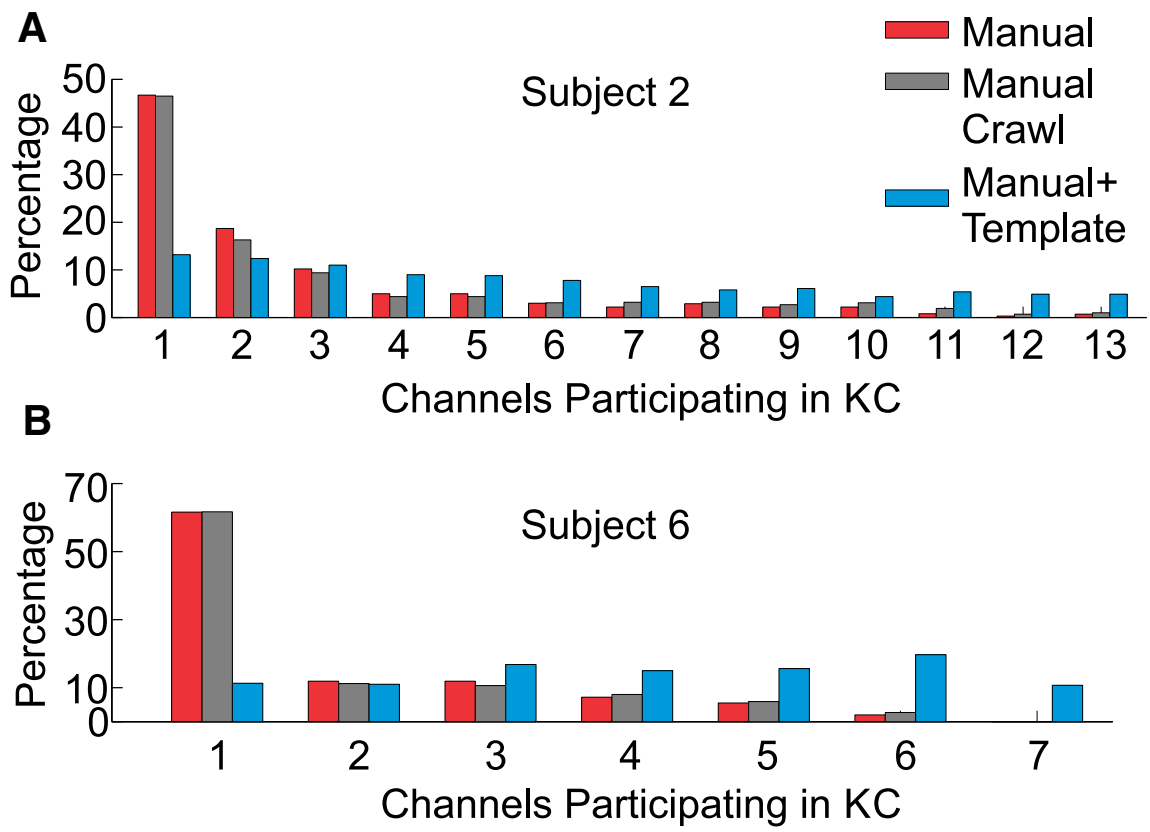

Figure 10. Including KC-like activity increases multichannel KC participation. The addition of KC-like activity (blue columns) to the manually marked KCs (red columns) increased the number of channels that were participating in KCs for all subjects. $\boldsymbol{A}$, In subjects where a small number of manually marked KCs included the participation of all channels, the addition of KC-like activity increased the percentage of such KCs. B, Subjects who did not show maximum channel participation with manually marked KCs displayed KCs that included all channels after the addition of KC-like activity. In subjects 2 and 6 (pictured), and subject 3 (not pictured), the classification of KCs using a 200 ms crawl window slightly shifted the distribution of manual multichannel participation to the right (gray columns), but not to the extent of the addition of KC-like activity (blue columns).

apparent lack of any systematic sequential order of KCs across the cortex. This was achieved by repeating the analysis described above after the addition of the template KCs, where a multinomial test was used to identify channel pairs with significant sequential ordering. Due to the addition of so many more KCs, a total of 412 pairs of channels (compared with 341 pairs in the manual-only $\mathrm{KCs}$ ) were tested over a representative night for all nine patients. Of these pairs, 33 pairs over four subjects were significant in addition to the pairs found using only manual KCs, for a total of 53 significant pairs (13\%). Subjects 1,4 , 8 , and 9 did not show any significant pairs before or after the addition of KC-like activity. A single significant pair, from ventral to dorsal, was found in subject 5 after the addition of KC-like activity. Subject 6 did not add any additional pairs.

Subject 3 was one of the subjects for whom anteriorto-posterior propagation could be most clearly tested due to electrode placement in prefrontal and parietal cortices (Fig. 7A). Of six prefrontal-parietal pairs examined, there were no significant pairs using only manual KCs, and, even after the addition of KC-like activity, there was no evidence of significant frontal-to-parietal propagation; rather, there were two significant pairs found within frontal cortex. Subject 7 did not have any significant manual pairs; however, one pair along the same electrode became significant after the addition of KC-like activity. Subject 2 already showed a large number of significant pairs (16) in testing manual KCs; the addition of KC-like activity added 29 significant pairs (Fig. 7B). This subject shows a strong tendency for significant pairs, in fact $64 \%$ of such pairs, to lead to temporal cortex. Apart from this pattern, the other pairs show sequences in many directions within and between hemispheres. In addition to the 47 significant pairs plotted in Figure 7, there are only 6 other significant pairs over three subjects, including both manual and KC-like activity, that were significant and have been described above but are not plotted. While the addition of KC-like activity makes it more possible to see evidence of anterior-to-posterior propagation, frontal-toparietal propagation still is not seen in the eight contact pairs in three subjects where it could be tested. Although propagation within the frontal lobe in various directions is seen, the only consistent propagation between lobes is from frontal to temporal.

\section{Insular origin of KCs is not supported by intracranial recordings}

In addition to the channel pairs that had a significant order of KC occurrence, we examined a specific prediction made in the literature regarding the typical origin of $\mathrm{KCs}$. Murphy et al. (2009) interpreted scalp EEG as demonstrating that $46 \%$ of isolated SOs (i.e. KCs) originate in the insula. Using bipolar SEEG, we demonstrated insular generation of KCs in two contacts in two patients. Of $1080 \mathrm{KC}$ events in these subjects, only $177(16 \%)$ involved the insula. Thus, at least $84 \%$ of $\mathrm{KC}$ events originated outside the insula. Of the $\mathrm{KC}$ events that did include the insula, not all began in the insula. Specifically, of the $61 \mathrm{KC}$ events involving the insula and at least one other structure, the 
insula led in $38 \mathrm{KCs}(62 \%)$. If we extrapolate this proportion to all insula KCs, then the proportion of all KCs led by the insula would be $\sim 10 \%$ (62\% of $16 \%$ ). While our sampling of two insular contacts from two patients is small, the inference of Murphy et al. (2009) does not appear to be supported by direct intracranial recordings.

\section{KCs may show a slight anterior-to-posterior propagation when all individual trials are tested}

In order to directly test the hypothesis that there is a significant distance versus delay relationship between anterior and posterior cortex during the $\mathrm{KC}$, we examined 6224 manual plus template-detected KCs that involved 51 pairs located along the anterior-to-posterior axis. To isolate anterior-to-posterior propagation from lateral-tomedial propagation, selected pairs could only have one contact separation in the medial-to-lateral direction. We found $3702 \mathrm{KC}$ pairs where the peak latency occurred first in the anterior channel followed by the posterior channel; in contrast, we found only $2518 \mathrm{KC}$ pairs that showed the opposite progression from the posterior to the anterior channel. This represents an $\sim 47 \%$ increase of KCs in the anterior-to-posterior direction compared with the posterior-to-anterior direction. The remaining four pairs exhibited a 0 ms delay. The distance between these channel pairs was plotted as a function of the average KC latency difference between them (Fig. 11A, black circles and red triangles). Distance between channel pairs was also plotted against the individual KC latency differences between them (Fig. 11B, black circles and red triangles). A linear mixed-model regression found that a significant relationship between anterior-to-posterior distance and delay existed across these pairs, considering either the average $\mathrm{KC}$ latency difference (Fig. 11A, purple line; $p=$ 0.007 ) or the individual KC latency difference (Fig. 11B, purple line; $p<10^{-4}$ ).

As we found that a large number of pairs in one subject exhibited a significant propagation from prefrontal areas to temporal cortex, we examined the effect of eliminating these pairs, which are plotted as red triangles in Figure 11. When these pairs are excluded, there are 2394 pairs that progress from anterior to posterior and 2061 pairs that progress from posterior to anterior, which only represents a $16 \%$ increase in anterior-to-posterior pairs. The black circles in Figure 11 represent the nontemporal pairs that were included in a subsequent linear mixed-model regression, which did not find a significant relationship between anterior-to-posterior distance and average KC latency differences (Fig. 11A, green line; $p=0.08$ ); however, when $\mathrm{KC}$ latency differences were considered individually, a significant relationship again occurred (Fig. 11B, green line; $p=0.003$ ).

In all cases, significant distance versus delay relationships explained only a small percentage of the variance: $2 \%$ when all pairs are considered individually, $13 \%$ when the average $\mathrm{KC}$ latency differences for all pairs are used, or $0.2 \%$ when individual pairs, excluding temporal pairs, are considered.

The above analyses considered the relative anteriorposterior location of the two contact pairs, in order to test the hypothesis that there is a global anterior-to-posterior propagation of KCs. This hypothesis predicts that anterior sites would precede posterior sites, and that the time delay would increase with increasing distance. We also tested the hypothesis that, regardless of direction, there is an overall relationship of distance versus delay, by examining the absolute distances and absolute delays of all pairs. As the distribution is no longer normal, a regression could not be calculated. Instead, the correlation calculated between the absolute distance and absolute delay of these KC pairs was very small $(r=0.071)$, although it was significant $(p=2.1 \mathrm{e}-08)$. As a direct comparison, the correlation was also calculated between the signed distance and absolute delay, as modeled above. This correlation was again significant, and greater than that for absolute distance and absolute delay, but it was still small $(r=0.12, p<2.2 \mathrm{e}-16)$.

In summary, there is a highly significant excess of KCs that propagate in the anterior-to-posterior direction compared with the posterior-to-anterior direction, and the average delays between locations are related to the distance between them, provided that the frontal-totemporal pairs are included. However, although this is true on a statistical basis, the variation between sites and individual KCs is very large, so this effect only explains a very small part of the observed pattern.

\section{Stage 2 and stage $3 \mathrm{KCs}$ have similar neurophysiological characteristics}

In the above analyses, KCs were defined by their morphology and accompanying decrease in high gamma power, and thus were not confined to stage 2 sleep. In order to compare NREM stage 2 sleep (N2) and N3 KCs, sleep staging was performed on scalp electrodes for the patients from Massachusetts General Hospital and on SEEG for the patients from La Timone Hospital, where scalp EEG was unavailable. Unless otherwise noted, all analyses presented below were performed on the combined manually detected and template-detected KCs. There were $2.8 \%$ fewer $\mathrm{KCs}$ added using template detection during N2 than N3, which was not significantly different when tested over all subjects using a paired $t$ test $(p=$ 0.64).

Averaged KC waveforms overlaid for N2 and N3 for the four channels of subject 1 have indistinguishable morphologies (Fig. 12A). To test whether there is a statistically significant difference between N2 and N3 peak amplitudes, $z$-scores for N2 and N3 were calculated for the KC peak amplitude for each channel with respect to the null values. When tested, $z$-scored amplitudes for N2 and N3 over all channels show no significant difference (paired $t$ test, $p=0.9$ ).

To evaluate whether a difference in the high gamma drop that characterizes the down state differs between N2 and N3, the Hilbert analytic amplitude for high gamma power was calculated from $25 \mathrm{~ms}$ before to $25 \mathrm{~ms}$ after the $\mathrm{KC}$ peak at $0 \mathrm{~ms}$ for all $\mathrm{KCs}$. These values were averaged over all KCs in a channel, separately for N2 and $\mathrm{N} 3$. A paired $t$ test between the averaged N2 and N3 high 


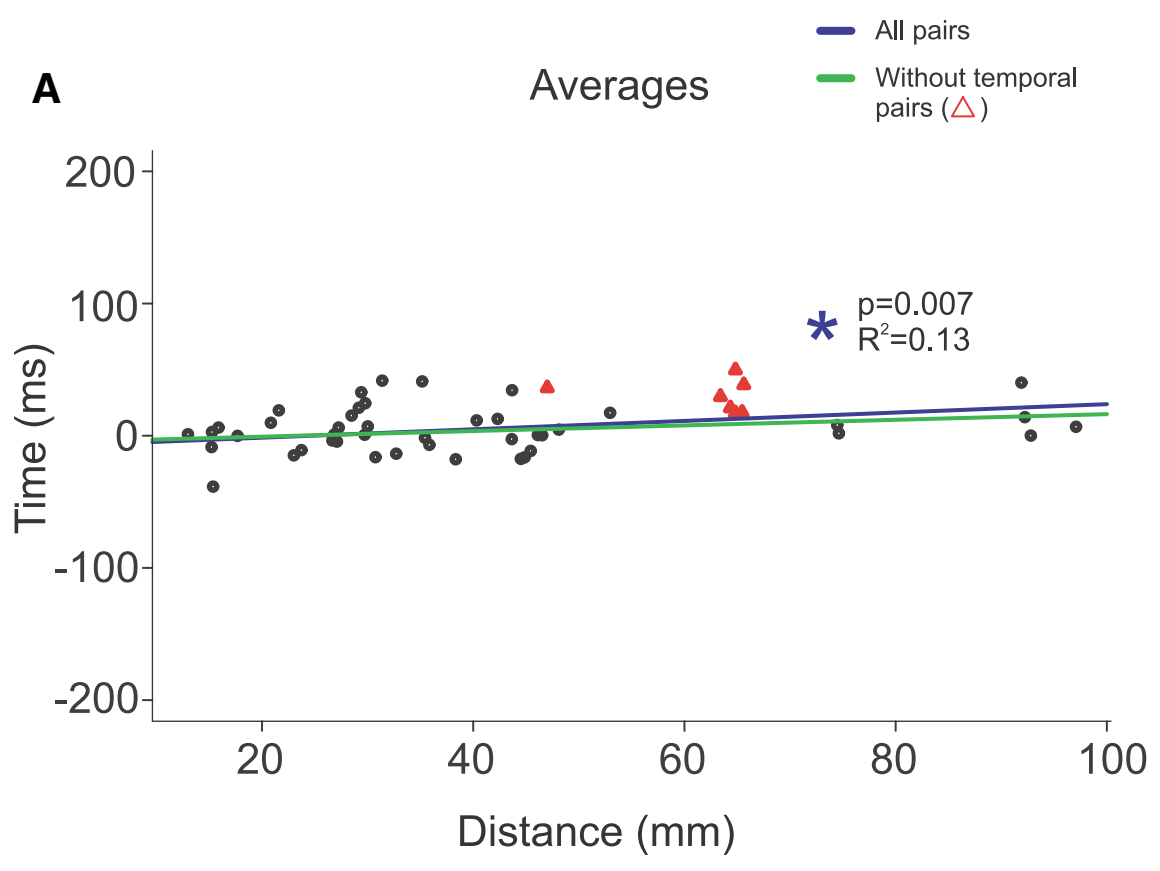

B Individual Trials

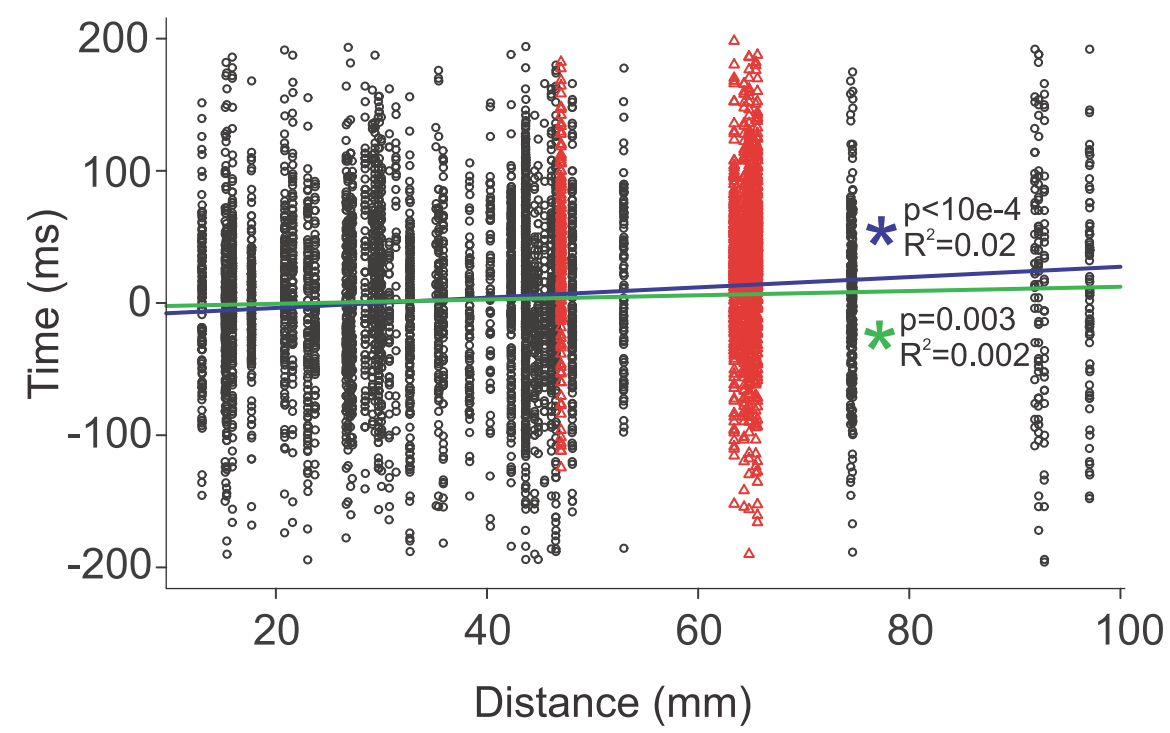

Figure 11. Anterior-to-posterior contact pair distance versus $\mathrm{KC}$ latency delay. $\boldsymbol{A}$, The distance between anterior-to-posterior channel pairs was plotted as a function of the average KC latency difference between the two channels, for all contact pairs (black circles and red triangles). The linear mixed-model regression between anterior-to-posterior distance and delay for all pairs is plotted as a purple line $(p=0.007)$, and, after excluding the temporal pairs (plotted with red triangles), is plotted as a green line $(p=0.08)$. $\boldsymbol{B}$, The distance between the same anterior-to-posterior channel pairs as in $\boldsymbol{A}$ was plotted as a function of each individual KC latency difference (black circles and red triangles). A linear mixed-model regression calculated to determine whether a significant relationship existed for individual KCs on these pairs was significant at $p<10^{-4}$ (purple line). Excluding the temporal pairs plotted with red triangles, the linear mixed-model regression was still significant at $p=0.003$ (green line).

gamma values over all channels found no significant difference $(p=0.34)$.

In order to determine whether manually marked N3 KCs more closely resemble a slow oscillation than $\mathrm{N} 2 \mathrm{KCs}$, the pre-KC peak amplitude was detected within a -850 to $-350 \mathrm{~ms}$ window prior to the $\mathrm{KC}$ peak at $0 \mathrm{~ms}$ and compared with the amplitude of this KC peak. Assuming that the up and down states of a slow oscillation have the same amplitude but opposite polarities, a slow oscillation would be implied if the pre-KC peak were equal to the $\mathrm{KC}$ peak. This was a more direct measure of whether the KC was preceded by a slow oscillation up state than the previously used pre-KC variance measure, which tested overall variability and was not scaled to the expected 
A

Subject 1
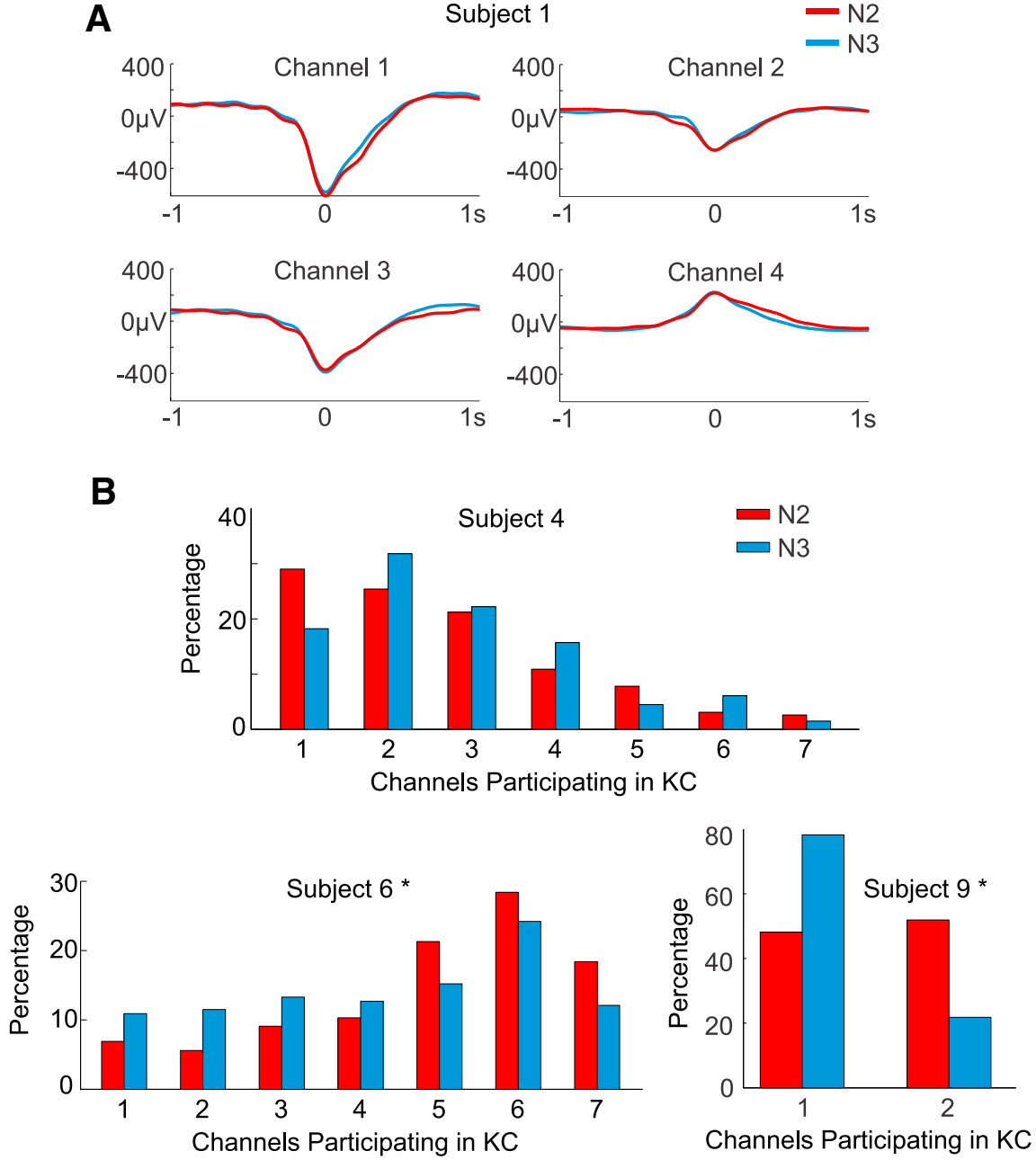

Figure 12. Comparison of N2 and N3 KCs. A, Waveforms of averaged N2 and N3 KCs overlaid for subject 1's four channels exhibit highly similar KC morphology. $\boldsymbol{B}$, Multichannel KC participation is not consistently greater in N2 or N3. The percentage of KCs occurring in each number of channels for N2 (red) and N3 (blue) are plotted for subjects 4, 6, and 9 . Subjects 6 and 9 show N2 KCs involving more channels, but this is not the pattern seen in all subjects, as shown with subject 4 . When a $\chi^{2}$ test was performed for each subject on these distributions, only subjects $6(p=0.011)$ and $9(p=0.000056)$ exhibited significant differences.

value of an SO. When the means over all KCs over all channels are calculated, the pre-KC peak of both manual $\mathrm{N} 2$ and $\mathrm{N} 3 \mathrm{KCs}$ is equal to $26 \%$ of the KC peak; in comparison, template-detected N2 KC means are $67 \%$, while template-detected N3 KC means are $83 \%$. If the mode is calculated instead, pre-KC amplitude of manually detected N2 KCs are $19 \%$ of the KC peak, compared with $22 \%$ for manually detected N3 KCs; again, this is much smaller than for the template-detected $\mathrm{KCs}$, which are $43 \%$ for N2 and $44 \%$ for N3. Therefore, the manually detected KCs showed similar pre-KC amplitudes regardless of sleep stage, which were much smaller than would be expected for a slow oscillation and smaller than template-detected KCs.

To test for a difference in the occurrence rate between $\mathrm{N} 2$ and N3, the average interstimulus (i.e. KC) interval for each stage for each channel was calculated after applying a Box-Cox transform to normalize the data. Of all 55 channels, 32 channels $(58 \%)$ occurred less frequently for
$\mathrm{N} 2$ than for N3. A one-tailed paired $t$ test found that N2 KCs occur less frequently than N3 KCs $(p=0.003)$. In summary, KCs in stage 2 and 3 NREM sleep have very similar morphologies, pre-KC amplitudes, KC peak amplitudes, and accompanying decreases in high gamma power. N3 KCs occurred more frequently than N2 KCs.

\section{KCs in N2 and N3 do not differ in the number of participating channels}

A separate $\chi^{2}$ test was performed for each subject, comparing the number of KCs occurring in N2 and N3 over each number of channels participating in a $\mathrm{KC}$ (i.e., one channel up to the maximum number of channels recorded for that subject). For the majority of subjects, there was no significant difference between N2 and N3 KCs in terms of multichannel participation, as follows: subject $1(p=$ $0.055)$; subject 2 ( $p=0.077)$; subject 3 ( $p=0.068)$; subject $4(p=0.061)$; subject $5(p=0.78)$; subject $7(p=$ $0.97)$; and subject $8(p=0.38)$. While some of these 
values are close to reaching significance, there is no clear pattern of one stage involving more or fewer channels than the other stage. As an example of this, subject 4 is presented in Figure 12B with the percentage of KCs occurring in each number of channels by stage, with N2 plotted in red and N3 in blue. For two subjects, there was a significant difference between the distribution of channels participating in N2 and N3 KCs [subject $6(p=0.011)$, subject $9(p=0.000056)]$. In both cases, the distribution of $\mathrm{N} 2 \mathrm{KCs}$ is skewed to the right, indicating that N2 KCs involve a greater number of channels than N3 KCs for that subject. The percentage of KCs occurring in each number of channels by stage for these two significant subjects is plotted in Figure 12B.

These results could indicate that there is a greater co-occurrence of a KC across locations during N2 compared with N3. Subject 9, however, only involved two channels, and therefore, it is difficult to predict whether this relationship would remain with the addition of more channels. The results for the other subjects that did not reach significance also exhibited variable patterns, some with slightly more multichannel KCs in N2, and others in N3, or neither. Thus, if there is a difference between stages in multichannel participation, it is weak.

\section{KCs in N2 and N3 do not differ significantly in their propagation sequences}

As described above, a significantly consistent direction of propagation between each pair of bipolar recordings was detected using binomial tests. Since the power of the binomial test is critically dependent on the number of observations, we subsampled the N2 or N3 data in any given patient so that the number of $\mathrm{KC}$ events was equal for the two sleep stages. With the much-reduced power, the number of significant pairs was only 39 for N2, and 27 for N3, without Bonferroni correction, of 180 bipolar pairs $\left(\chi^{2}=2.67, \mathrm{df}=1, p>0.10\right)$. Due to the small number of remaining events after balancing $\mathrm{N} 2$ and N3 in each subject, we are unable to draw any strong conclusions regarding whether the patterns of sequences are significantly different in N2 or N3.

In summary, the similarities and differences between $\mathrm{N} 2$ and N3 KCs support the hypothesis that physiological and morphological properties define the $\mathrm{KC}$, rather than their occurrence during a particular stage. For the majority of subjects, there was no difference in multichannel participation between stages, while findings regarding propagation patterns were inconclusive. We conclude that the defining physiological and morphological characteristics of a KC do not differ between N2 and N3, and therefore, that global KC properties may be studied collapsed between stages.

\section{Summary of current results}

Figure 13 depicts a summary of our findings in contrast to current views. Based on scalp EEG and MEG, as well as referential intracranial recordings, a monolithic interpretation of KCs (or their equivalent isolated SOs) has been put forth wherein $\mathrm{KCs}$ are generated initially and most strongly in prefrontal cortex (Fig. 13A, orange star), and then propagate consistently to parietal cortex (Fig. 13A,
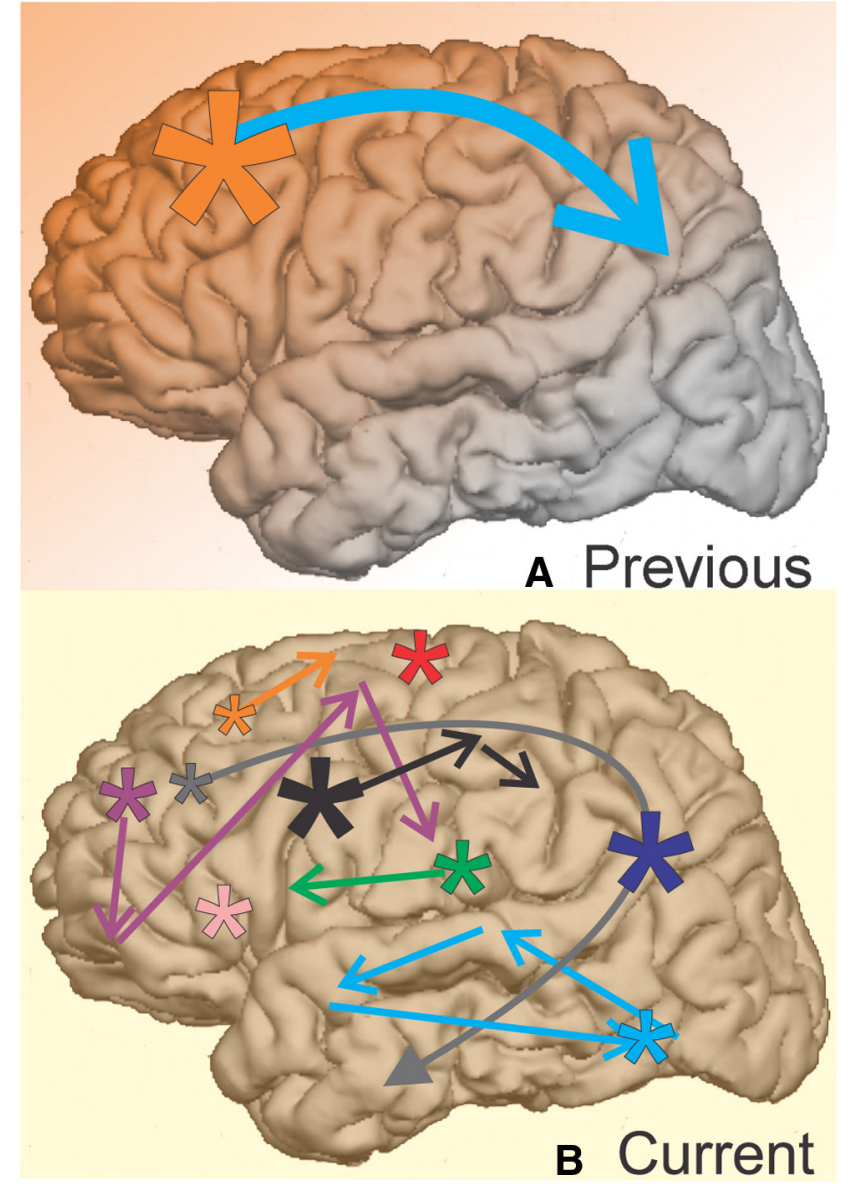

Figure 13. Summary of current $\mathrm{KC}$ findings compared with previous views. A, Previous scalp EEG and intracranial studies of the $\mathrm{KC}$ and isolated SOs (i.e., KCs) find the KCs with the largest amplitudes in midline prefrontal regions (orange star), as well as strong (blue arrow), one-directional (faded orange background) propagation from anterior to posterior cortical regions. B, Our current findings indicate that when measured locally, KCs are actually smallest in amplitude in anterior prefrontal regions (orange star). KCs may co-occur (uniform yellow background) across variably small or large areas of cortex (stars and arrows). Propagation may occur from anterior to temporal regions (gray arrow), but also occurs in a variety of patterns across the cortex (groups of colored arrows). This variability in $\mathrm{KC}$ onset, extent, and spread could be used by the cortex during replay to consolidate memories encoded with correspondingly variable spatiotemporal encoding patterns.

blue arrow and fading orange background). In contrast, our results demonstrate that $\mathrm{KCs}$ are smallest in anterior prefrontal areas (Fig. 13B, orange star), that they arise all over the cortex (additional stars), and that they may cooccur across many areas, as represented by the uniform yellow background. While there is a weak overall tendency for KCs to propagate posteriorly, especially from frontal to temporal sites (gray arrow), KCs seldom exhibit a consistent pattern of propagation between any two cortical locations (groups of colored arrows). Furthermore, the current results show that while some KCs involve widespread cortical locations, others can be very 
focal. In summary, our results suggest that each KC may be unique in its origin, extent, and spread.

\section{Discussion}

Although KCs have been studied for over 80 years, their origin and spread remain controversial. Do they occur quasi-synchronously in widespread cortical areas (MakMcCully et al., 2014), or arise in midline frontal areas and spread parietally (Massimini et al., 2004)? Are KCs a fundamental cortical process, or are some cortical areas unable to generate KCs (Wennberg, 2010)? In locations generating $\mathrm{KCs}$, are their amplitudes and occurrence rates uniform? These issues remain controversial because previous studies used methods that provide ambiguous localization. We examined the spatial and temporal relationships of individual KCs recorded simultaneously in multiple cortical locations using bipolar SEEG recordings, validated with HGP. Using this unambiguous method, we found that KCs can be generated throughout the cortex, including areas previously asserted not to generate KCs: the cingulate, ventral temporal, and occipital cortices. Contrary to previous accounts, KCs were smallest in anterior prefrontal areas. A given KC may occur in only one sampled location, or may co-occur in widespread cortical sites. When KCs were absent in other channels, KC-like activity was often found. Regardless of whether this KClike activity was included, most channel pairs did not show a regular propagation direction. However, there was a weak statistical tendency favoring anterior-to-posterior propagation. We conclude that KCs have multiple degrees of freedom that may support the spatiotemporal patterns underlying memory consolidation and cortical restoration.

\section{Methodology for obtaining unambiguous generator localization}

Previous examinations of $\mathrm{KCs}$ in humans used $\mathrm{EEG}$, MEG, EEG with fMRI, ECOG, or referential SEEG. In contrast, our study used bipolar SEEG derivations, where one contact was immediately above the local cortical surface, and one was in the immediately subjacent white matter. We validated KCs by demonstrating an accompanying statistically significant drop in HGP. Thus, unlike previous studies, we could be certain of local generation at the electrode site.

Dense microelectrode array recordings demonstrate that the $\mathrm{KC}$ is a cortical down state, with a cessation of neuronal firing and a broadband decrease in spectral power of the local field potential (LFP), due to inward apical dendritic currents in layers $2 / 3$ (Cash et al., 2009). The current return is in layer 1 , resulting in a surface negativity in ECOG or SEEG. Ambiguities arise because each sensor records the summation and cancelation of all cortical potentials. Although distant areas contribute to the LFP inversely according to the square of their distance (Lindén et al., 2011), their total contribution may exceed that of local sources when much of the cortex is active (Klee and Rall, 1977).

All potential measurements are relative to a reference electrode, and the above comments assume an inactive reference. However, when generators are distributed, it is difficult to confirm that a reference electrode location is inactive. Extracranial references cannot be assumed to be inactive because the amplitude of distributed generators declines little from cortex to scalp (Cooper et al., 1965). Average references are not neutral unless all sides of the volume conductor are equally and densely sampled, a practical impossibility due to the neck and face, and to scalp incisions. Thus, even locally recorded potentials with ECOG and SEEG are difficult to interpret as definitively reflecting only locally generated activity.

Bipolar transcortical SEEG derivations are insensitive to distant sources because the two contacts act as references for each other. The positive and negative peaks of the locally generated $\mathrm{KC}$ are determined by the separation between the generating transmembrane inward and outward currents, measured to be separated by $\sim 1 \mathrm{~mm}$ (Cash et al., 2009). The two leads in the bipolar SEEG pair were separated by $2.6-5.6 \mathrm{~mm}$, enabling them to capture the entire local field. In contrast, volume-conducted potentials from distant generators change little between the leads, and thus are removed by recording the difference between the leads. In addition, our KCs were accompanied by a suppression of broadband HGP, which has been observed to be highly focal (Lachaux et al., 2005), due to asynchronous generators (Lindén et al., 2011). Thus, the co-occurrence of HGP suppression provides a strong confirmation of local KC generation.

The ambiguities resulting from the superposition of volume conducted potentials from many locations is much more severe for extracranial (scalp) EEG, where all locations are distant and the intervening skull and subcranial CSF further spread the potential (Ahlfors et al., 2010; Irimia et al., 2012). Inverse solutions use the pattern of potentials across sensors to infer possible generators. However, the inverse problem is mathematically ill posed unless unproven assumptions are made. Scalp EEG also has a reference that is likely to be active. MEG does not use a reference, and is not smeared by the skull or CSF, but it also suffers from an irretrievably ambiguous inverse problem due to superposition of multiple sources at the sensors (Liu et al., 2002). While PET scanning and fMRI provide unambiguous localization, and can be triggered by $\mathrm{KC}$ s recorded by simultaneous EEG, they lack the temporal resolution to determine whether the activity they locate is due to the KC itself or to other time-locked activity.

Slow oscillations, and to a limited extent, KCs have also been examined in animals. SOs occur synchronously over long distances in anaesthetized (Volgushev et al., 2006) and naturally sleeping cats (Destexhe et al., 1999), as do KCs (Amzica and Steriade, 1998). SOs are also synchronous in anesthetized rats (Isomura et al., 2006). In contrast, SOs in ferret slices propagate slowly (SanchezVives and McCormick, 2000). Besides the effects of anesthesia and the loss of synaptic connections in slices, the brain volume of humans is $\sim 750$ larger than rats and $\sim 45$ times larger than cats (Haug, 1987), making the problem of synchronization or propagation different.

In summary, the recordings reported here better characterize KC origin and spread because they were less 
prone to the ambiguities inherent in previous methods. However, electrodes were placed for localizing seizures in patients with long-standing epilepsy. Epilepsy can be caused by many factors, including a widespread genetic alteration in excitability that could affect the occurrence, amplitude, or spread of KCs. Conversely, the epilepsies of the patients studied here were localized and nongenetic. Rarely, KCs have been noted to be altered in partial epilepsy: KC rate increases, and elevated amplitude KClike phenomena precede some seizures in refractory nocturnal frontal lobe epilepsy (Si et al., 2010); however, none of the patients analyzed here have this type of epilepsy. $\mathrm{KC}$-like phenomena have occasionally been associated with epileptiform spikes in temporal lobe epilepsy (Geyer et al., 2006), but would have been identified and eliminated from the KCs studied here. Furthermore, we minimized the possible confounding effects of epileptiform activity by excluding all contacts in regions implicated in the subject's epilepsy, and all epochs showing any signs of epileptic activity. Nonetheless, we cannot completely exclude the possibility that the patient's epilepsy affected our results.

Since clinical considerations dictated electrode placement, we were also limited in the cortical areas sampled, which differed in number and location between patients. Recordings also differed in electrode contact spacing and the geometric relationship of the contact to the local anatomy. This variability was addressed with linear mixed-effects models, which assume no interaction between patient and electrode location. If a patient's epilepsy type were to differentially influence the size of KCs in different locations, then this assumption would be violated. Overall, dense sampling in a larger number of patients would further disentangle the multiple influences on $\mathrm{KC}$ origin, amplitude, occurrence, and spread.

\section{KC generator localization}

Previous studies of KC generation have yielded a variety of localizations. Inverse solutions applied to MEG or scalp EEG estimate KC generators to deep locations in the parietal lobe (Lu et al., 1992), the sylvian fissure (Iramina and Ueno, 1996), midline structures (Murphy et al., 2009; Wennberg and Cheyne, 2013), or deep in the parietal lobe or other variable deep locations (Numminen et al., 1996). These results are often inconsistent with each other and, overall, with our results. For example, consistent with our findings, Wennberg and Cheyne (2013) estimated KC generators to subcallosal or cingulate cortices. However, they are only two of many generators, EEG and MEG estimates did not agree, and other generators localized in this study were clearly incorrect, as they acknowledge. Murphy et al. (2009) estimated the scalp EEG isolated SO down state (i.e., KC) generators mainly to the anterior cingulate, with secondary inferior frontal gyrus, precuneus, and insular generators, and no significant generation in many other locations, which we demonstrate to generate KCs. The most parsimonious solution for most inverse methods when applied to widespread generators is to estimate a relatively strong but focal deep source.
Tones that evoke KCs also evoke increased BOLD in the superior temporal plane and inferior frontal gyrus; pars opercularis (Czisch et al., 2009; Dang-Vu et al., 2011). However, these increases are likely to represent BOLD activity related to the tone, rather than the $\mathrm{KC}$, which is a down state. During SOs, PET scanning also shows a superior temporal increase, with a subcallosal decrease (Dang-Vu et al., 2005). While the PET scan findings are during SOs, their location corresponds with some of the areas where we demonstrated $\mathrm{KC}$ generation.

Wennberg (2010) investigated KC localization using average reference ECOG and SEEG values in humans, and recorded the $\mathrm{KCs}$ with the largest amplitude in dorsal frontal areas, which he interpreted as being the site of the largest KC generators. We demonstrate how average reference SEEG may detect KCs in locations where they are not generated. In these sites, KCs are not seen in bipolar SEEG, and they do not correspond to a local drop in HGP. Thus, the presence or absence of KCs in referential SEEG must be interpreted with caution. In contrast, we found that KCs in anterior prefrontal regions are small and infrequent, while $\mathrm{KCs}$ occur more frequently in posterior prefrontal cortex and posterior cingulate. We also demonstrate that $\mathrm{KCs}$ are generated in the cingulate, ventral temporal, and occipital cortices, where Wennberg (2010) concluded that KCs are not generated based on his failure to record them. Our data regarding ventral temporal sites are not definitive because they were located in regions implicated in epileptogenesis. The emphasis of Wennberg (2010) on dorsolateral prefrontal generators may also have partially arisen from his averaging of SEEG KCs at their frontal scalp EEG peak, while we identified KCs individually at each cortical site.

Nir et al. (2011) demonstrated that unit firing is correlated with SOs recorded in the medial superior frontal gyrus, precuneus, anterior and posterior cingulate, entorhinal cortex, and parahippocampal gyrus, thus demonstrating local generation. Although not specifically reported, their data suggest that these locations also generate KCs. Using linear microelectrode arrays, Cash et al. (2009) reported local transmembrane currents during KCs in prefrontal, lateral orbital, middle and superior temporal, and supramarginal gyri. Using the same method, Csercsa et al. (2010) demonstrated local generation of SO in the postcentral and prefrontal cortices. These studies are consistent with our finding of locally generated KCs in prefrontal, medioventral temporal, cingulate, orbital, and supramarginal cortices, and demonstrate or imply generation in the superior temporal and postcentral gyri, and the precuneus, where we failed to record. Overall, our findings, combined with those of earlier studies, demonstrate very widespread KC generation.

This conclusion is inconsistent with the studies described above, which have been interpreted as indicating that large areas of cortex do not generate KCs. Using the more definitive methods described here, every location that was adequately sampled generated KCs, implying that KCs may represent a fundamental operating mode of human cortex. This conclusion raises the question of why KCs show significantly different amplitudes in different 
cortical locations. A complete answer cannot be given until the precise circuit and channel mechanisms of $\mathrm{KC}$ generation are better understood. However, different cortical areas vary considerably in their levels of particular ligand- and voltage-gated channels, and associated proteins (Zilles et al., 2002; Hawrylycz et al.,2012), in particular, $\mathrm{GABA}_{\mathrm{B}}$ and potassium channels, which may be involved in KC generation (Cunningham et al., 2006; Kohl and Paulsen, 2010).

\section{KC propagation}

Prior to this study, there had not been a rigorous examination of how KCs may propagate across the human cortex. In an influential study, Massimini et al. (2004) proposed that scalp EEG SOs in more posterior scalp sites peaked progressively later, with a delay of $\sim 200 \mathrm{~ms}$, or a mean propagation speed of $2.5 \mathrm{~m} / \mathrm{s}$. This view was extended by Murphy et al. (2009) in their analysis of isolated spontaneous SOs (i.e., KCs). Wennberg (2010), however, noted that KCs appeared to occur synchronously; when tested explicity (Mak-McCully et al., 2014), the observed delays in the peaks of averaged KCs between anterior and posterior sites in EEG, ECOG, or SEEG were $\sim 10$ times faster than had been previously predicted for isolated SOs (Massimini et al., 2004; Murphy et al., 2009). Since averaging could obscure propagation patterns, we systematically tested here for such patterns in single KCs. Again, we found scant evidence for regular propagation patterns. When KCs occurred on different bipolar contacts within $200 \mathrm{~ms}$ of each other, they were tested to determine whether their order of occurrence was significantly different from chance. This occurred in only $6 \%$ of manually detected $\mathrm{KCs}$, or $13 \%$ when templatedetected KCs are also included. Several cases of significant propagation were observed within the frontal lobe, including along the cingulate, where propagation could be in any direction. Such complex patterns of propagation across the cortex have previously been noted by Hangya et al. (2011). Frontal-to-parietal propagation, however, occurred in none of the eight pairs of bipolar contacts where it could be tested. A preponderance of insular origins of isolated SOs (i.e., KCs; Murphy et al., 2009) was also not supported by our data in the two patients in whom it could be tested. The most common pattern of propagation was from lateral prefrontal to lateral temporal, which is reminiscent of the regular SO propagation from medial frontoparietal to medial temporal lobe locations found by Nir et al. (2011), who also failed to find regular propagation within the medial frontoparietal sites. SO propagation from anterior to temporal regions for both medial and lateral cortex was also found by Botella-Soler et al. (2012).

Notwithstanding these negative results, we found a highly significant excess of $\mathrm{KCs}$ that propagate in the anterior-to-posterior direction compared with the reverse, and that average delays between locations are related to their spatial separation. Although this is true on a statistical basis, the variation between sites and individual KCs is large, so this effect explains only $2 \%$ of the observed pattern.

\section{KCs are not confined to stage 2}

Sleep stages defined using scalp EEG do not necessarily indicate a uniform and synchronous participation by different brain areas. For example, sleep onset measured locally in the thalamus has been noted to occur before sleep onset is detected in scalp EEG (Caderas et al., 1982; Gücer et al., 1978) or locally in the cortex (Magnin et al., 2010). We have also observed that isolated KCs may occur in parietal areas, while rhythmic slow oscillations occur in frontal areas. Thus, we rigorously detected locally generated KCs using LFPs and HGP, and then characterized them regardless of sleep stage. Post hoc analyses showed that pre-KC amplitude, KC peak amplitude, and high gamma characteristics did not differ between stages 2 and 3. However, KCs did occur more frequently in N3 than N2. Critically, the extent of KC cortical involvement does not appear to change between stages.

\section{Functional implications of KC heterogeneity}

In summary, this is the first study to not only characterize the local generation of KCs in human cortex, but to examine their variable amplitude and occurrence rate, cooccurrence across the cortex, and propagation (Fig. 13). Although systematic propagation of KCs between cortical sites can be observed, these are rare, and, in most cases, the patterns of propagation vary randomly from $\mathrm{KC}$ to $\mathrm{KC}$. Similarly, although there is a slight overall tendency for more anterior sites to lead more posterior ones, this tendency only explains a minute proportion of the variance, again leading to the conclusion that successive KCs are highly heterogeneous. We show in this article that KCs differ greatly not only in their propagation patterns, but also in which areas are engaged, even though all areas seem to generate some KC activity. It has previously been shown that KCs can be universal and quasi-synchronous across widespread cortical sites (Mak-McCully et al., 2014). Here, we confirm this finding and show that KCs can also be highly focal or intermediate. This conclusion was robust to different methods of clustering KCs across channels, and was strengthened when using templateselected as well as manually selected KCs.

$\mathrm{KC}$ heterogeneity may arise from different corticocortical or cortico-thalamic mechanisms underlying the $\mathrm{KC}$. Recent work has re-examined the role of the thalamus in the production of SOs, which have long been thought to be an exclusively cortico-cortico mechanism, and found the thalamus to be important for SO frequency (David et al., 2013) and synchrony (Lemieux et al., 2014). Additionally, disruption of spindling in the thalamus has been posited as a mechanism of quasi-synchronous KC generation (Mak-McCully et al., 2014). Variable involvement of thalamic versus cortical mechanisms may help to account for variability in the location and spread of KCs.

$\mathrm{KC}$ heterogeneity may also reflect differential engagement of different cortical areas during the preceding waking period (Huber et al., 2004). This modulation of $\mathrm{KC}$ distribution may reflect restorative or consolidation processes during sleep. The later function is supported by the correlation of increasing SOs with 
behavioral consolidation (Marshall et al., 2006). In rodents, KCs are associated with the replay of firing patterns in the hippocampus and neocortex ( $\mathrm{Ji}$ and Wilson, 2007). The previous conception of KCs as monolithic in origin, extent, and spread would offer little scope for KCs to support the consolidation of distinct memories. In contrast, our demonstration that individual KCs are highly variable in location and propagation could provide a substrate for memory consolidation processes to engage different cortical networks, or the same networks in different sequences.

\section{References}

Ahlfors SP, Han J, Lin FH, Witzel T, Belliveau JW, Hämäläinen MS, Halgren E (2010) Cancellation of EEG and MEG signals generated by extended and distributed sources. Hum Brain Mapp 31:140149. CrossRef Medline

Amzica F, Steriade M (1998) Cellular substrates and laminar profile of sleep K-complex. Neuroscience 82:671-686. Medline

Botella-Soler V, Valderrama M, Crépon B, Navarro V, Le Van Quyen M (2012) Large-scale cortical dynamics of sleep slow waves. PLoS One 7:e30757. CrossRef Medline

Box GEP, Cox DR (1964) An analysis of transformations (with discussion). J R Stat Soc B 26:211-252.

Caderas M, Niedermeyer E, Uematsu S, Long DM, Nastalski J (1982) Sleep spindles recorded from deep cerebral structures in man. Clin Electroencephalogr 13:216-225. Medline

Cash SS, Halgren E, Dehghani N, Rossetti AO, Thesen T, Wang C, Devinsky O, Kuzniecky R, Doyle W, Madsen JR, Bromfield E, Eross L, Halász P, Karmos G, Csercsa R, Wittner L, Ulbert I (2009) The human K-complex represents an isolated cortical down-state. Science 324:1084-1087. CrossRef Medline[Mismatch]

Colrain IM (2005) The K-complex: a 7-decade history. Sleep 28:255273. Medline

Cooper R, Winther AL, Crow HJ, Walter WG (1965) Comparison of subcortical, cortical and scalp activity using chronically indwelling electrodes in man. Electroencephalogr Clin Neurophysiol 18:217228. Medline [Mismatch]

Csercsa R, Dombovári B, Fabó D, Wittner L, Eross L, Entz L, Sólyom A, Rásonyi G, Szucs A, Kelemen A, Jakus R, Juhos V, Grand L, Magony A, Halász P, Freund TF, Maglóczky Z, Cash SS, Papp L, Karmos G, et al. (2010) Laminar analysis of slow wave activity in humans. Brain 133:2814-2829. CrossRef Medline

Cunningham MO, Pervouchine DD, Racca C, Kopell NJ, Davies CH, Jones RS, Traub RD, Whittington MA (2006) Neuronal metabolism governs cortical network response state. Proc Natl Acad Sci U S A 103:5597-5601. CrossRef Medline

Czisch M, Wehrle R, Stiegler A, Peters H, Andrade K, Holsboer F, Sämann PG (2009) Acoustic oddball during NREM sleep: a combined EEG/fMRI study. PLoS One 4:e6749. CrossRef Medline[Mismatch]

Dang-Vu TT, Desseilles M, Laureys S, Degueldre C, Perrin F, Phillips C, Maquet P, Peigneux P (2005) Cerebral correlates of delta waves during non-REM sleep revisited. Neuroimage 28:14-21. CrossRef Medline

Dang-Vu TT, Bonjean M, Schabus M, Boly M, Darsaud A, Desseilles M, Degueldre C, Balteau E, Phillips C, Luxen A, Sejnowski TJ, Maquet $P$ (2011) Interplay between spontaneous and induced brain activity during human non-rapid eye movement sleep. Proc Natl Acad Sci U S A 108:15438-15443. CrossRef Medline

David F, Schmiedt JT, Taylor HL, Orban G, Di Giovanni G, Uebele VN, Renger JJ, Lambert RC, Leresche N, Crunelli V (2013) Essential thalamic contribution to slow waves of natural sleep. J Neurosci 33:19599-19610. CrossRef Medline

Delorme A, Makeig S (2004) EEGLAB: an open source toolbox for analysis of single-trial EEG dynamics including independent component analysis. J Neurosci Methods 134:9-21. CrossRef Medline
Destexhe A, Contreras D, Steriade M (1999) Spatiotemporal analysis of local field potentials and unit discharges in cat cerebral cortex during natural wake and sleep states. J Neurosci 19:4595-4608.

Destrieux C, Fischl B, Dale A, Halgren E (2010) Automatic parcellation of human cortical gyri and sulci using standard anatomical nomenclature. Neurolmage 53:1-15. CrossRef Medline

Diekelmann S, Born J (2010) The memory function of sleep. Nat Rev Neurosci 11:114-126. CrossRef Medline

Dykstra AR, Chan AM, Quinn BT, Zepeda R, Keller CJ, Cormier J, Madsen JR, Eskandar EN, Cash SS (2012) Individualized localization and cortical surface-based registration of intracranial electrodes. Neuroimage 59:3563-3570. CrossRef Medline

Geyer JD, Carney PR, Gilliam F (2006) Focal epileptiform spikes in conjuction with K-complexes. J Clin Neurophysiol 23:436-439. CrossRef

Gücer G, Niedermeyer E, Long DM (1978) Thalamic EEG recordings in patients with chronic pain. J Neurol 219:47-61. Medline

Halász P (2005) K-complex, a reactive EEG graphoelement of NREM sleep: an old chap in a new garment. Sleep Med Rev 9:391-412. CrossRef

Hangya B, Tihanyi BT, Entz L, Fabó D, Eróss L, Wittner L, Jakus R, Varga V, Freund TF, Ulbert I (2011) Complex propagation patterns characterize human cortical activity during slow-wave sleep. $J$ Neurosci 31:8770-8779. CrossRef

Haug H (1987) Brain sizes, surfaces, and neuronal sizes of the cortex cerebri: a stereological investigation of man and his variability and a comparison with some mammals (primates, whales, marsupials, insectivores, and one elephant). Am J Anat 180:126-142. CrossRef

Hawrylycz MJ, Lein ES, Guillozet-Bongaarts AL, Shen $E H, N g ~ L$, Miller JA, van de Lagemaat LN, Smith KA, Ebbert A, Riley ZL, Abajian C, Beckmann CF, Bernard A, Bertagnolli D, Boe AF, Cartagena PM, Chakravarty MM, Chapin M, Chong J, Dalley RA, et al. (2012) An anatomically comprehensive atlas of the adult human brain transcriptome. Nature 489:391-399. CrossRef Medline

Huber R, Ghilardi MF, Massimini M, Tononi G (2004) Local sleep and learning. Nature 430:78-81. CrossRef Medline

Iramina K, Ueno S (1996) Source estimation of spontaneous MEG activity and auditory evoked responses in normal subjects during sleep. Brain Topogr 8:297-301. Medline

Irimia A, Van Horn JD, Halgren E (2012) Source cancellation profiles of electroencephalography and magnetoencephalography. Neuroimage 59:2464-2474. CrossRef Medline

Isomura Y, Sirota A, Ozen S, Montgomery S, Mizuseki K, Henze DA, Buzsáki G (2006) Integration and segregation of activity in entorhinal-hippocampal subregions by neocortical slow oscillations. Neuron 52:871-882. CrossRef Medline

Ji D, Wilson MA (2007) Coordinated memory replay in the visual cortex and hippocampus during sleep. Nat Neurosci 10:100-107. CrossRef Medline

Klee M, Rall W (1977) Computed potentials of cortically arranged populations of neurons. J Neurophysiol 40:647-666. Medline

Kohl MM, Paulsen O (2010) The roles of GABAB receptors in cortical network activity. Adv Pharmacol 58:205-229. CrossRef Medline

Lachaux JP, George N, Tallon-Baudry C, Martinerie J, Hugueville L, Minotti L, Kahane P, Renault B (2005) The many faces of the gamma band response to complex visual stimuli. Neurolmage 25:491-501. CrossRef Medline

Lemieux M, Chen JY, Lonjers P, Bazhenov M, Timofeev I (2014) The impact of cortical deafferentation on the neocortical slow oscillation. J Neurosci 34:5689-5703. CrossRef Medline

Lindén H, Tetzlaff T, Potjans TC, Pettersen KH, Grün S, Diesmann M, Einevoll GT (2011) Modeling the spatial reach of the LFP. Neuron 72:859-872. CrossRef Medline

Liu AK, Dale AM, Belliveau JW (2002) Monte Carlo simulation studies of EEG and MEG localization accuracy. Hum Brain Mapp 16:4762. Medline

Lu ST, Kajola M, Joutsiniemi SL, Knuutila J, Hari R (1992) Generator sites of spontaneous MEG activity during sleep. Electroencephalogr Clin Neurophysiol 82:182-196. Medline 
Magnin $M$, Rey $M$, Bastuji $H$, Guillemant $P$, Mauguière $F$, GarciaLarrea $L$ (2010) Thalamic deactivation at sleep onset precedes that of the cerebral cortex in humans. Proc Natl Acad Sci U S A 107:3829-3833. CrossRef Medline

Mak-McCully RA, Deiss SR, Rosen BQ, Jung KY, Sejnowski TJ, Bastuji H, Rey M, Cash SS, Bazhenov M, Halgren E (2014) Synchronization of isolated downstates (K-complexes) may be caused by cortically-induced disruption of thalamic spindling. PLoS Comput Biol 10:e1003855. CrossRef Medline

Marshall L, Helgadóttir H, Mölle M, Born J (2006) Boosting slow oscillations during sleep potentiates memory. Nature 444:610613. CrossRef Medline

Massimini M, Huber R, Ferrarelli F, Hill S, Tononi G (2004) The sleep slow oscillation as a traveling wave. J Neurosci 24:6862-6870. CrossRef Medline

MATLAB (2009) version 7.9.0 (R2009b). Natick, MA: MathWorks.

Murphy M, Riedner BA, Huber R, Massimini M, Ferrarelli F, Tononi G (2009) Source modeling sleep slow waves. Proc Natl Acad Sci U S A 106:1608-1613. CrossRef Medline

Nakagawa S, Schielzeth H (2013) A general and simple method for obtaining R2 from generalized linear mixed-effects models. Methods Ecol Evol 4:133-142. CrossRef

Nir Y, Staba RJ, Andrillon T, Vyazovskiy VV, Cirelli C, Fried I, Tononi G (2011) Regional slow waves and spindles in human sleep. Neuron 70:153-169. CrossRef Medline

Numminen J, Mäkelä JP, Hari R (1996) Distributions and sources of magnetoencephalographic K-complexes. Electroencephalogr Clin Neurophysiol 99:544-555. Medline

Oostenveld R, Fries P, Maris E, Schoffelen JM (2011) FieldTrip: open source software for advanced analysis of MEG, EEG, and invasive electrophysiological data. Comput Intell Neurosci 2011:156869. CrossRef Medline

Pinheiro J, Bates D, DebRoy S, Sarkar D, R Core Team (2015) nlme: linear and nonlinear mixed effects models. In: R package version 3.1-120 Edition. Vienna, Austria: R Foundation for Statistical Computing.
R Development Core Team (2004) R: a language and environment for statistical computing. Vienna, Austria: R Foundation for Statistical Computing.

Riedner BA, Hulse BK, Murphy MJ, Ferrarelli F, Tononi G (2011) Temporal dynamics of cortical sources underlying spontaneous and peripherally evoked slow waves. Prog Brain Res 193:201-218. CrossRef Medline

Sanchez-Vives MV, McCormick DA (2000) Cellular and network mechanisms of rhythmic recurrent activity in neocortex. Nat Neurosci 3:1027-1034. CrossRef Medline

Si Y, Liu L, Li Q, Mu J, Tian LY, Chen JN, Zhou D (2010) Features of the K-complex waves in refractory nocturnal frontal lobe epilepsy. Epilepsy Res 92:219-225. CrossRef Medline

Silber MH, Ancoli-Israel S, Bonnet MH, Chokroverty S, GriggDamberger MM, Hirshkowitz M, Kapen S, Keenan SA, Kryger MH, Penzel T, Pressman MR, Iber C (2007) The visual scoring of sleep in adults. J Clin Sleep Med 3:121-131. Medline

Tononi G, Cirelli C (2014) Sleep and the price of plasticity: from synaptic and cellular homeostasis to memory consolidation and integration. Neuron 81:12-34. CrossRef Medline

Volgushev M, Chauvette S, Mukovski M, Timofeev I (2006) Precise long-range synchronization of activity and silence in neocortical neurons during slow-wave oscillations [corrected]. J Neurosci 26: 5665-5672. CrossRef Medline

Wennberg R (2010) Intracranial cortical localization of the human K-complex. Clin Neurophysiol 121:1176-1186. CrossRef [Mismatch]

Wennberg R, Cheyne D (2013) On noninvasive source imaging of the human K-complex. Clin Neurophysiol 124:941-955. CrossRef Medline

Zilles K, Palomero-Gallagher N, Grefkes C, Scheperjans F, Boy C, Amunts $\mathrm{K}$, Schleicher A (2002) Architectonics of the human cerebral cortex and transmitter receptor fingerprints: reconciling functional neuroanatomy and neurochemistry. Eur Neuropsychopharmacol 12:587-599. CrossRef 